\title{
Nanoscale
}

\section{Breathing new life into old antibiotics: Overcoming antibacterial resistance by antibiotic-loaded nanogel carriers with cationic surface functionality}

Received 00th January 20xx Accepted 00th January 20xx

DOI: $10.1039 / x 0 x x 00000 x$

www.rsc.org/

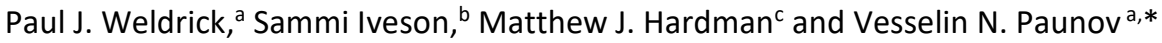

\begin{abstract}
Multidrug-resistant pathogens are prevalent in chronic wounds. There is an urgent need to develop novel antimicrobials and formulation strategies that can overcome antibiotic resistance, and provide a safe alternative to traditional antibiotics. This work aimed to develop a novel nanocarrier for two cationic antibiotics, tetracycline and lincomycin hydrochloride which can potentially overcome antibiotic resistance. In this study, we report the use of surface functionalised polyacrylic copolymer nanogels as carriers for cationic antibiotics. These nanogels can encapsulate small cationic antimicrobial molecules and act as a drug delivery system. They were further functionalised with a biocompatible cationic polyelectrolyte, bPEI, to increase their affinity towards the negatively charged bacterial cell walls. These bPEI-coated nanocarrierencapsulated antibiotics were assessed against a range of wound isolated pathogens, which had been shown through antimicrobial susceptibility testing (AST) to be resistant to tetracycline and lincomycin. Our data reveal that bPEI-coated nanogels with encapsulated tetracycline or lincomycin displayed increased antimicrobial performance against selected wound-derived bacteria, including strains highly resistant to the free antibiotic in solution. Additionally, our nanocarrierbased antibiotics showed no detectable cytotoxic effect against human keratinocytes. We attribute the increase in antimicrobial activity of the cationically-functionalised antibiotic-loaded nanogel carriers to specific electrostatic adhesion to the microbial cell wall delivering a higher local antibiotic concentration, confirmed by scanning electron microscopy. Such nanotechnology based approach may enhance the effectiveness of a wide variety of existing antibiotics, offering a potentially new mechanism to overcome antibiotic resistance.
\end{abstract}

\section{Introduction}

Antibiotics are bactericidal or bacteriostatic to microorganisms, removing them from the body, or inhibiting them sufficiently that the host immune system can fight infection without being out competed by fast-growing microorganisms. ${ }^{1}$ Antibiotics can function by inhibiting the synthesis of the cell wall, synthesis of DNA, RNA, or proteins. They enter the cell by diffusing through the cell wall/membrane, or by energy dependent transport mechanisms. ${ }^{2,3}$ However, antibiotic resistance emerged almost immediately after penicillin was used, and resistance species where already discovered before penicillin first began to be administered to patients. ${ }^{4}$ This emergence of resistance has been greatly amplified by the mis- and over-prescription of antibiotics. ${ }^{5}$ The lack of recent antibiotic discovery has tipped the balance of discovery/resistance towards bacteria, with an ever-increasing population of resistant species. ${ }^{6,7}$ Modes of resistance which bacteria may employ include; expression efflux pumps which create an extracellular gradient reducing the concentration of the antibiotic in the cell; enzymatic inactivation of

\footnotetext{
a. Department of Chemistry and Biochemistry, University of Hull, Hull, HU6 7RX, UK.

b. Department of Biomedical Sciences, University of Hull, Hull, HU6 7RX, UK.

c. Centre for Atherothrombosis and Metabolic Disease, Hull York Medical School, Hull, HU6 7RX, UK.

*Corresponding author: Tel: +44 1482 465660; E-mail: v.n.paunov@hull.ac.uk. Electronic Supplementary Information (ESI) available: [details of any supplementary information available should be included here]. See DOI: 10.1039/x0xx00000x
}

the antibiotic; decreased membrane permeability; or modification of the target to reduce antibiotic affinity. ${ }^{8} \mathrm{~S}$. aureus has been studied extensively regarding antibiotic resistance, within six years of aminoglycoside introduction, resistance had already emerged via enzyme modification, with over 50 separate enzymes discovered. ${ }^{9}$ When penicillinase-producing $S$. aureus was targeted with a semisynthetic analogous penicillin drug, methicillin, this soon became inhibited leading to MRSA strains. ${ }^{10} \mathrm{~A}$ similar pattern occurred with vancomycin resistance (VRSA) in 2002, with multi-drug resistance becoming an increasing obstacle. ${ }^{11,12}$ Spread of resistance is increased by bacterial ability to exchange genetic material. ${ }^{13}$

Tetracycline is a broad-spectrum antibiotic, active against many clinically relevant pathogens. ${ }^{14}$ There are species such as $P$. aeruginosa, which have an intrinsic resistance due to the presence of multi-drug efflux transporters, and endogenous antimicrobial inactivation. ${ }^{15}$ Tetracycline inhibits protein synthesis by blocking the ribosomal site $A$, reducing the ability of charged aminoacyl-tRNA to enter the active site. ${ }^{16}$ Bacteria can commonly become resistant to tetracycline by the horizontal gene transfer of efflux pumps. ${ }^{17}$ Lincomycin is a member of the lincosamide antibiotic group and was first used clinically in $1964 .{ }^{18}$ It demonstrates strong activity against Gram-positive species, but weaker activity against some Gramnegative species. ${ }^{19}$ Similar to tetracycline, lincomycin prevents protein synthesis. ${ }^{20}$ However, lincomycin binds to the $23 \mathrm{~S}$ portion of the $50 \mathrm{~S}$ subunit of bacterial ribosomes causing the dissociation of peptidyl-tRNA. ${ }^{21}$ Bacteria can resist lincomycin by the use of multidrug efflux pumps. ${ }^{22}$ 
A
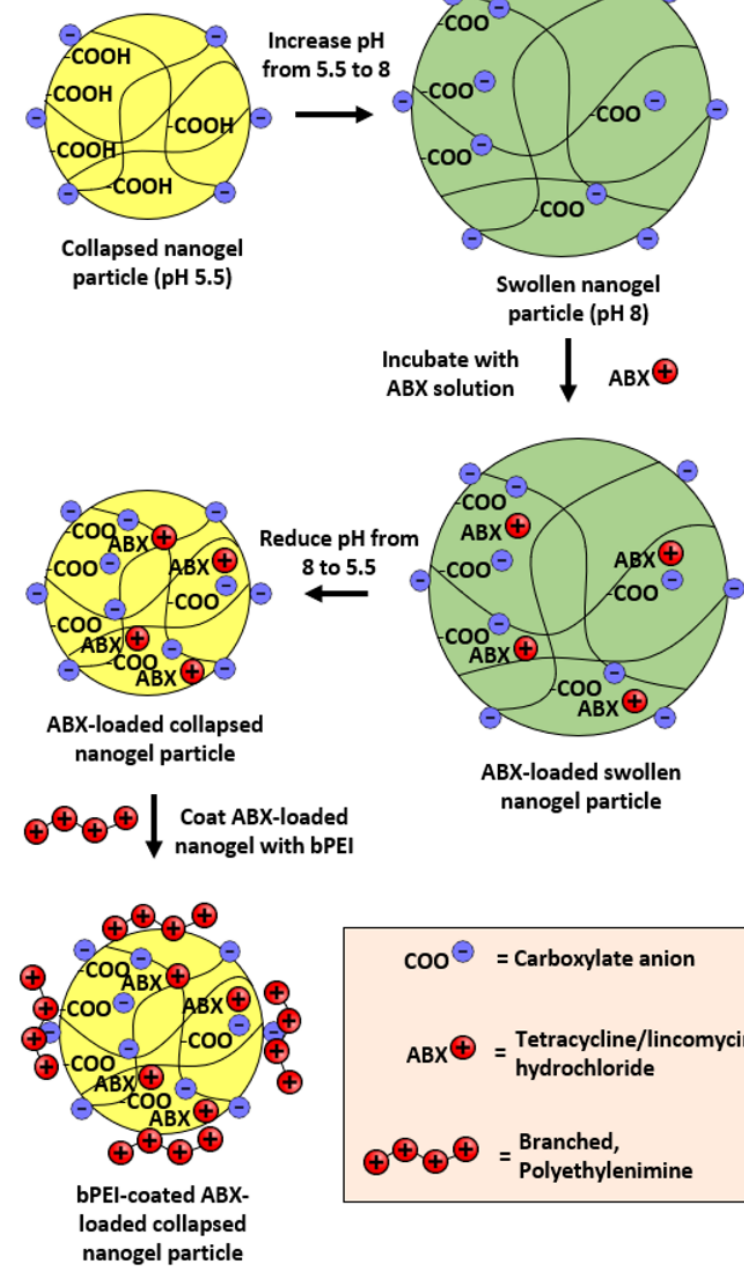
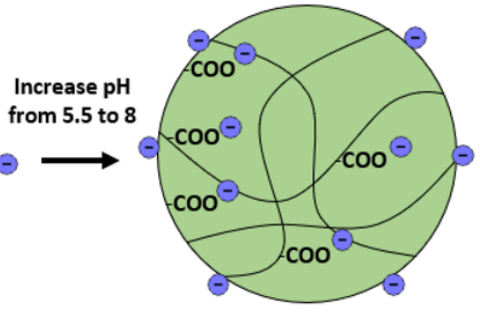

Swollen nanogel particle (pH 8)

cubate with

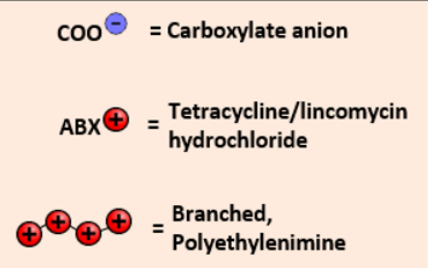

B<smiles>CC(C)CC(C(=O)O)C(C)(C)C</smiles>

Polyacrylic acid

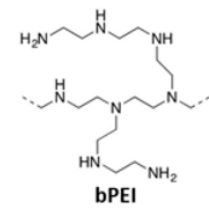

(polyethylenimine,

brached)

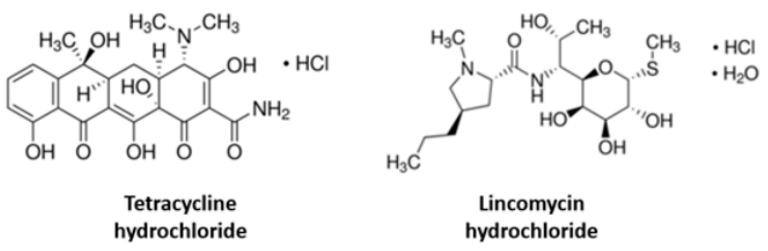

C
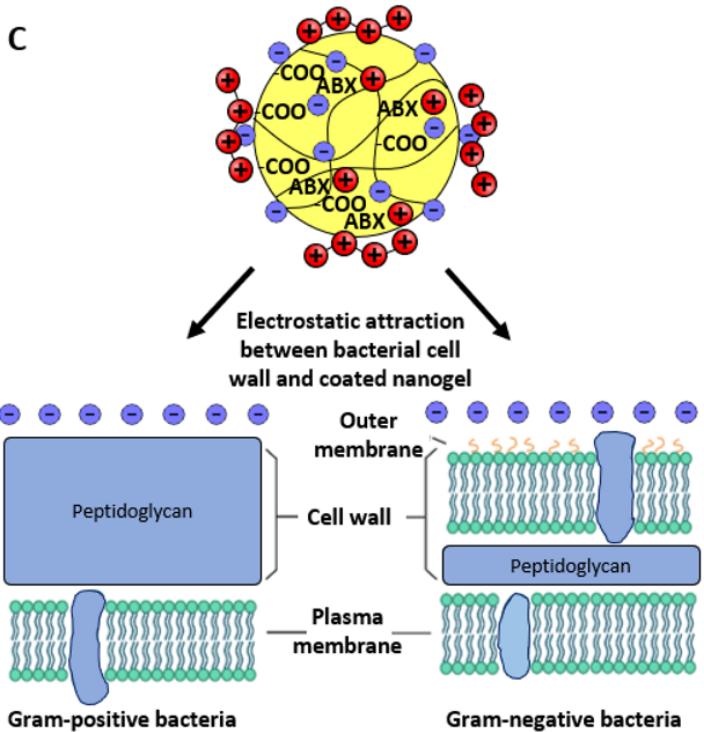

Figure 1. (A) A schematic diagram of the swelling $(\mathrm{pH} 8)$ and deswelling $(\mathrm{pH} 5.5)$ method $^{6}$ used to encapsulate tetracycline and lincomycin hydrochlorides into a polyacrylic acid nanocarrier (Carbopol Aqua SF1). A biocompatible cationic polyelectrolyte (bPEI) was used to functionalise the nanocarrier surface which makes it non-toxic for mammalian cells, (B) The chemical formulas of polyacrylic acid (in the nanogel - partially cross-linked), bPEl, tetracycline hydrochloride, and lincomycin hydrochloride. (C) The mechanism for the delivery of antibiotics to the surface of bacteria. The positive charge pf the nanoparticle due to ionisation of the bPEI coating binds to the negatively charged surface groups of the bacterial cell wall. The nanoparticle acts as a drug delivery vehicle for cationic antibiotics allowing a localised and continuously release, which can potentially overwhelm the microorganism resistance mechanisms.

Previous attempts have been made to utilise antibiotics in a way which reduces the development of resistant strains. ${ }^{35}$ Sequential regimens alternating between two or more antibiotics doses at sublethal concentrations has been shown to reduce collateral sensitivity and help preserve antimicrobial susceptibility to secondary antibiotic treatments, helping to limit the emergence of resistant species..$^{23,36,37}$ Synergistic antibiotic paired treatments are often more effective than single treatments. Novel synergistic combinations are rare, but have been found using high-throughput genetic dataset analysis of bacteria exposed to different antibiotics. ${ }^{24}$ Efforts have also been made in inhibiting the mRNA encoding for efflux pumps expressed in resistant bacteria. Anti-sense oligonucleotides have been shown to inhibit mRNA translation resulting in the reduced expression of efflux pumps, enabling once resisted antibiotics to be effective again. ${ }^{25}$ Recently, Geilich et al. ${ }^{38}$ reported a polymerosome based carrier of silver nanoparticles which show promising results against antibiotic resistant bacteria. Despite progress in previous research there still remains an urgent need for overcoming resistance in pathogens using a method which is non-invasive and simple to use in antimicrobial formulations.

Nanocarriers have previously been used to deliver drugs in a way which is target specific, increasing the concentration of the drug at the desired location. ${ }^{26}$ This can also reduce the unwanted interaction of the drug in healthy tissue, facilitating a general reduced toxic effect. ${ }^{27}$ They can also protect the encapsulated active agent from possible degradation or inactivation within a biological system. ${ }^{28}$ 
Nanocarrier's must be biocompatible, biodegradable, nonimmunogenic, and not intrinsically toxic. ${ }^{29}$ Various strategies for using nanomaterials as antimicrobials and delivery of antibiotics have been recently reviewed. ${ }^{40}$ Tetracycline itself, being a cationic antibiotic has previously been encapsulated into poly(acrylic acid) based interpenetrating polymer gels (IPNs), ${ }^{41}$ however, these are not nanocarriers and did not include any bacterial targeting strategy. Nanogels are lightly-crosslinked ionisable polymer chains that can swell in aqueous solutions when mediated by $\mathrm{pH}$ changes. Commonly, polymers with carboxyl groups functionality are used to swell the nanogel cross-linked scaffold, but other groups such as sulphate and hydroxyl can be used. ${ }^{30}$ Polyacrylic nanogels have previously been used to encapsulate and deliver cationic antimicrobial molecules such as berberine hydrochloride and chlorhexidine di-gluconate. ${ }^{31,32}$ dual-functionalised shellac nanoparticles showed a boost in berberine antimicrobial effect. ${ }^{33}$ In the present study, we test a novel method of tetracycline and lincomycin delivery, designed to significantly enhance their antibiotic action on resistant bacteria. Specifically, these cationic antibiotics were encapsulated into the cores of a commercially available polyacrylic acid copolymer based nanogel, Carbopol Aqua SF1. The surface of the nanogel particles was further functionalised with a mid-molecular weight $\left(\mathrm{Mw}^{\sim 25}\right.$,000) biocompatible polyelectrolyte, branched polyethylenimine (bPEl; outlined in Figure $1 \mathrm{~A}$ ). We studied the antimicrobial efficacy of these antibiotic delivery vehicles against validated tetracycline and lincomycin resistant wound derived clinical isolates. The cationic antibiotic is encapsulated within the interior of the nanogel where it electrostatically interacts with partially dissociated $\mathrm{COOH}$ groups of the polyacrylic acid-based nanogel. Due to the cationic nature of the bPEI coating, the modified nanogel particles can adhere electrostatically to the negatively charged bacterial cell walls which allows the encapsulated antibiotic to be released in a very close proximity to the targeted bacteria with the purpose of amplifying its antimicrobial effect.

Gram-positive bacteria contain an abundance of teichoic acids, which are linked to either the peptidoglycan or the cell membrane directly. These teichoic acids facilitate a negative charge due the presence of phosphate in their molecular structure. Similarly, Gram-negative bacteria contain an outer layer of lipopolysaccharides and phospholipids, which confers a negative charge to the bacteria. ${ }^{39}$ This process is illustrated in Figure 1C. This strategy can potentially deliver much higher dose of antibiotic directly on the bacteria cell wall that can be achieved through oral intake and could temporarily overwhelm the bacteria efflux transporters. Additionally, targeted nanocarrier-based antibiotic delivery could avoid cytotoxicity by reducing the overall concentration of antibiotic required for efficacy. Here, for the first time we test this concept on a range of resistant and susceptible bacterial species clinically isolated from wound samples, in attempt to overcome antibiotic resistance without increasing the overall concentration of the antibiotic treatment.

\section{Materials and Methods}

\section{Materials}

Carbopol Aqua SF1 nanogel was supplied by Lubrizol, USA, as an aqueous suspension (30 wt\%). Polyethylenimine, branched (bPEI) was supplied by Sigma-Aldrich (99\%), Germany. Resazurin sodium salt (75\%, Sigma-Aldrich, UK) was used for cell metabolic activity assays and diluted in Dulbecco's Phosphate Buffered Saline (DPBS) (Gibco, Fisher Scientific, UK) at a concentration of $15 \mathrm{mg} \mathrm{mL}^{-1}$. CellTiter $96^{\circledR}$ AQueous One Solution Reagent was purchased from Promega, UK and used to measure the metabolic activity of $\mathrm{HaCaT}$ cells. Tetracycline hydrochloride and lincomycin hydrochloride were supplied by Sigma-Aldrich, UK. Mueller-Hilton Broth (MHB), MuellerHilton Agar (MHA), tetracycline $(30 \mu \mathrm{g})$ and lincomycin $(15 \mu \mathrm{g})$ Antimicrobial Susceptibility Disk were supplied by Oxoid, UK. DeyEngley neutralising broth was provided by Sigma-Aldrich, UK. Dubecco's Modified Eagle Medium, heated activated Foetal Bovine Serum (FBS), and L-Glutamine were delivered by Gibco (Fisher Scientific, UK). Trypsin (10X) was purchased from Sigma-Aldrick, UK) and Trypan Blue Solution ( $0.4 \%$ phosphate buffered saline was supplied by HyClone ${ }^{\mathrm{TM}}$ (GE Healthcare Sciences, UK). Staphylococcus aureus subsp. aureus Rosenbach (ATCC ${ }^{\circledR} 29213^{\mathrm{TM}}$ ) and Pseudomonas aeruginosa (Schroeter) Migula (ATCC ${ }^{\circ} 27853^{\text {Tm}}$ ) were purchased from ATCC. Wound isolated bacterial species (Table 1 ) were obtained from Hull Royal Infirmary, inoculated on bijoux slopes. Microbank bead cryovials (Pro-Lab Diagnostics, Canada) were used to store all species long-term at $-80{ }^{\circ} \mathrm{C}$ according to the manufacturer's protocol. Deionized water purified by reverse osmosis and ion exchange from a Milli-Q water system (Millipore, UK or PUR1TE SELECT, UK) was used in all our studies. Consumable plasticware used in the study were purchased from Sarstedt (UK), Thermo Scientific (UK), or CytoOne (UK) unless otherwise stated.

\section{Bacterial Culture}

Bacterial stock plates were created by streaking one Microbank bead onto an MHA plate and incubating for 24 hours at $37^{\circ} \mathrm{C}$ to produce viable colonies. Overnight $(\mathrm{O} / \mathrm{N})$ cultures were prepared by incubating a single colony scraped from the MHA stock plates into 10 $\mathrm{mL}$ of $\mathrm{MHB}$ for 16 hours at $37^{\circ} \mathrm{C}$ with $140 \mathrm{rpm}$ shaking (Labnet 211DS shaking incubator, Labnet International). For all bacterial assays, $\mathrm{O} / \mathrm{N}$ cultures were adjusted to $0.5 \mathrm{McF}$ arland standard by diluting the $\mathrm{O} / \mathrm{N}$ culture into $0.85 \mathrm{w} / \mathrm{v} \%$ serial saline until an optical density of 0.08 0.12 at $625 \mathrm{~nm}$ was obtained using a spectrophotometer (Jenway 7310, Cole-Parmer). These adjusted bacterial saline suspensions were then diluted 1:150 into MHB to yield starting concentrations between $5 \times 10^{5}-1 \times 10^{6}$ colony forming units per $\mathrm{mL}^{(\mathrm{CFU} \mathrm{mL}} \mathrm{m}^{-1}$ ).

Table 1. Clinical isolate bacteria used in this study.

\begin{tabular}{ll}
\hline Genus/Species & Origin \\
\hline Staphylococcus epidermidis & Leg ulcer \\
Staphylococcus aureus (methicillin-resistant) & Leg ulcer \\
Staphylococcus aureus & Sebaceous cyst \\
Pseudomonas aeruginosa & Leg ulcer \\
Enterococcus faecalis & Ulcer \\
Staphylococcus pseudointermedius & Burn \\
\hline
\end{tabular}



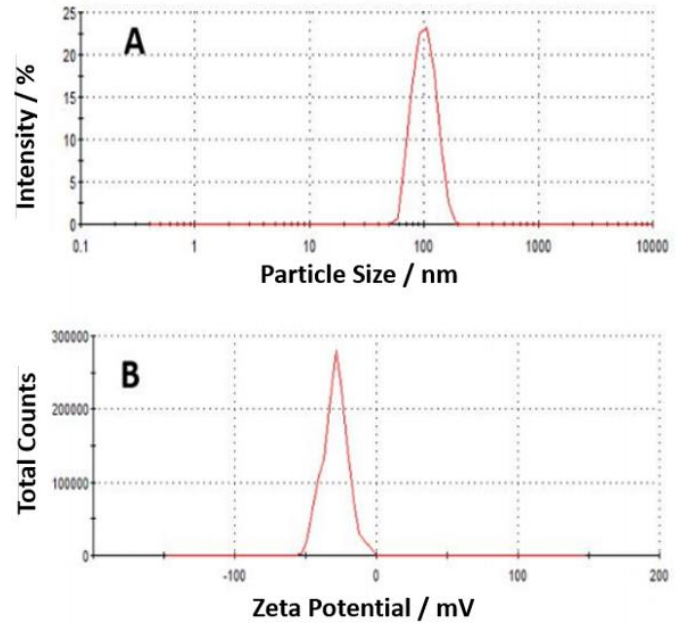
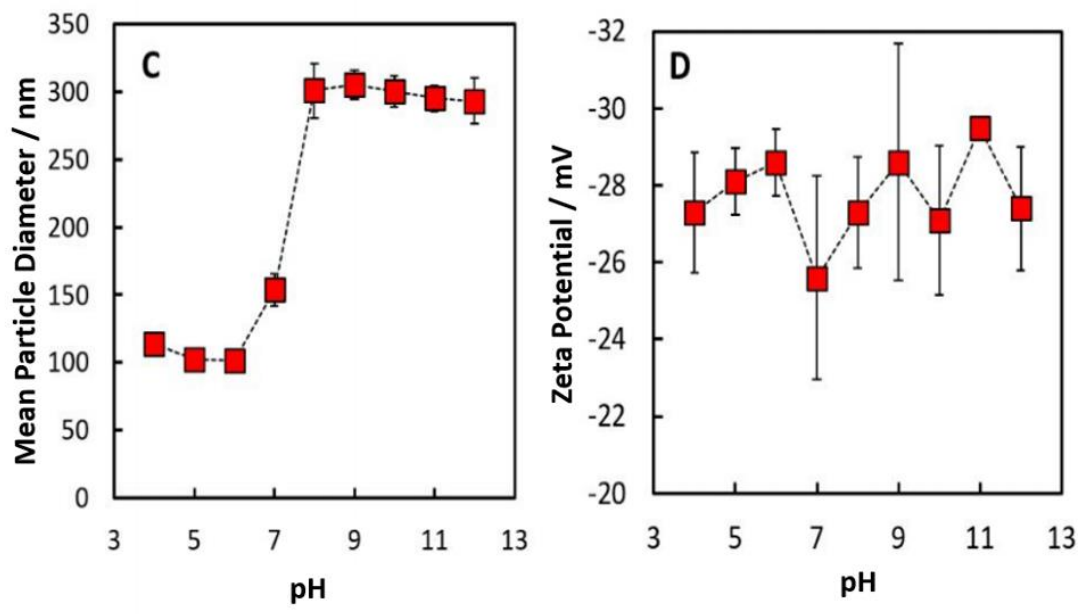

Figure 2. The nanogel particle size (A) and zeta potential (B) distribution of $0.02 \mathrm{wt} \%$ Carbopol Aqua solution was the diameter measured at pH 5 with a Zetasizer. (C) The 0.02 wt\% Carbopol Aqua SF1 nanogel mean particle hydrodynamic diameter and (D) mean zeta potential at varying pH. Particle size and zeta potential is measured using a DLS. Carbopol Aqua SF-1 was diluted from a $30 \mathrm{wt} \%$ stock with two refractive indexes (RI). pH 4-7 (collapsed nanogel) is measured with RI 1.450 and $\mathrm{pH} 8-12$ (swollen nanogel) with RI 1.336 at $25^{\circ} \mathrm{C}$. Each value represents a triple replicate with \pm S.D. The lines are guides to the eye.

\section{Encapsulation of tetracycline and lincomycin into Carbopol Aqua SF1 nanogel and its effect on Carbopol stability}

The principle of the encapsulating a cationic antibiotic (as a hydrochloride salt) is based on the swelling and deswelling cycle of the Carbopol Aqua SF1 at different pH. ${ }^{31}$ Briefly, a $0.2 \mathrm{wt} \%$ aqueous dispersion of the nanogel was prepared by adding $6.66 \mu \mathrm{L}$ of the 30 wt\% stock solution to a beaker and diluting in $10 \mathrm{~mL}$ of deionized water. This was then adjusted to $\mathrm{pH} 8$ by adding droplets of $0.25 \mathrm{M}$ $\mathrm{NaOH}$ whilst been stirred. The dispersion was then warmed to $37^{\circ} \mathrm{C}$. An aliquot of $0.2 \mathrm{wt} \%$ tetracycline/lincomycin aqueous dispersion was prepared by weighing $20 \mathrm{mg}$ of the antibiotic $(A B X=$ tetracycline or lincomycin) powder and diluting into $10 \mathrm{~mL}$ of deionized water and then warming to $37^{\circ} \mathrm{C}$. The $10 \mathrm{~mL}$ antibiotic solution was then added to the $\mathrm{pH} 8$ nanogel dispersion and shaken for 30 minutes at $37^{\circ} \mathrm{C}$ to allow the antibiotic to diffuse into and electrostatically bind to the swollen Carbopol nanogel. The $\mathrm{pH}$ of the ABX-Carbopol solution was then reduced to $\mathrm{pH} 5$ using droplets of $0.25 \mathrm{M} \mathrm{HCl}$ whilst being stirred for another 30 minutes. The ABX-Carbopol solution was then centrifuged at $4000 \mathrm{rpm}$ for 15 minutes, the supernatant removed and retained for encapsulation efficiency analysis. The pellet was washed twice with deionized water and re-dispersed into $10 \mathrm{~mL}$ of deionized water. The $\mathrm{pH}$ was then increased to 8 by gradually adding droplets of $0.25 \mathrm{M} \mathrm{NaOH}$ and the solution was gently stirred overnight. The final ABX-Carbopol solution was reduced to $\mathrm{pH} 5.5$ using acetate buffer solution. The particle size and zeta potential distribution of the ATX-Carbopol dispersion was measured using a Malvern Mastersizer as described above.

\section{Encapsulation efficiency of tetracycline and lincomycin in Carbopol Aqua SF1}

The encapsulation efficiency of $A B X$ was measured by analysing the supernatant collected from the centrifugation of ABX-Carbopol dispersion. The absorbance of the supernatant was measured at 625 $\mathrm{nm}$ using a spectrophotometer (Jenway 7310, Cole-Parmer).
Calibration curves of both tetracycline and lincomycin were produced by diluting different weights of the antibiotic into $10 \mathrm{~mL}$ of deionized water to create a range of aqueous solutions of $A B X$ of concentration in the range of $0-0.2 \mathrm{wt} \%$. A calibration graph was prepared for the absorbance as a function of the $A B X$ concentration. A linear regression was used to determine to the unknown concentration of the un-encapsulated $A B X$ in the supernatant. The encapsulation efficiency of the antibiotic loaded into the Carbopol Aqua SF1 was calculated as follows

$$
\text { Ecapsulation efficiency }(\%)=\frac{\text { total antibiotic }- \text { uncapsulated antibiotic }}{\text { total antibiotic }} \times 100 \text {. }
$$

\section{Release kinetics of tetracycline and lincomycin from Carbopol Aqua SF1 nanogel formulations}

The release kinetics of the $A B X$ from the Carbopol Aqua SF1 was measured by placing the $10 \mathrm{~mL} 0.2 \mathrm{wt} \%$ ABX-Carbopol solution into 12-14 kDa dialysis tubing (Serva, Germany) and sealed with plastic clips. The filled dialysis tubing was then placed into 500 $\mathrm{mL}$ of (i) an acetate buffer solution at $\mathrm{pH} 5.5$ or (ii) a phosphate buffer at $\mathrm{pH} 7.5$ in order to monitor the amount of released $A B X$ at a specific $\mathrm{pH}$ over 24 hours at room temperature. $1 \mathrm{~mL}$ aliquots of the acetate or phosphate buffer solution was removed at specific time points and the absorbance measured at $625 \mathrm{~nm}$ using a spectrophotometer (Jenway 7310, ColeParmer). $1 \mathrm{~mL}$ of either acetate of phosphate buffer solution was added to the respective solution to retain the volume at $500 \mathrm{~mL}$. Calibration curves of both tetracycline and lincomycin were created by diluting different weights of the $A B X$ into 500 $\mathrm{mL}$ of either acetate or phosphate buffer solutions to create a range of buffered solutions of $A B X$ of concentration between 0 and $0.2 \mathrm{wt} \%$. The amount of $A B X$ released from the Carbopol Aqua SF1 nanogel which has transferred into the buffer solution through the dialysis membrane pores was determined from the calibration curves. The percentage released $A B X$ leaching from the nanogel was calculated by the equation: 
$\mathrm{ABX}$ release $(\%)=\frac{\{\text { amount of } \mathrm{ABX} \text { in the buffer solution at a specific time }\}}{\{\text { amount of } \mathrm{ABX} \text { loaded into the nanogel }\}} \times 100$.

\section{Functionalisation of ABX-Carbopol Aqua SF1 nanogel with bPEI}

bPEI is a branched cationic polyelectrolyte which was used to reverse the surface charge of the ABX-Carbopol Aqua SF1 nanogel particles from negative to positive. A $0.005 \mathrm{wt} \% \mathrm{bPEI}$ aqueous solution was produced by weighing a $0.5 \mathrm{mg}$ aliquot of bPEI and placing into a beaker and dispersing in a total volume of $10 \mathrm{~mL}$ of deionized water whilst stirring. $10 \mathrm{~mL} 0.005$ wt\% bPEl aliquot was rapidly added to the $10 \mathrm{~mL} 0.2$ wt\% ABX-0.2 wt\% Carbopol dispersion with vigorous shaking for 5 minutes. The resulting mixture was then centrifuged at $4000 \mathrm{rpm}$ for 15 minutes. The supernatant was discarded, and the pellet re-dispersed into $10 \mathrm{~mL}$ of deionized water yielding a 0.005 wt\% bPEI-0.2 wt\% ABX-0.2 wt\% Carbopol Aqua SF1 dispersion which was used as a stock for antimicrobial testing against wound associated bacterial pathogens.

\section{Antimicrobial susceptibility testing (AST) Disk Diffusion Test of wound species}

A single colony of each species was isolated and placed into $10 \mathrm{~mL}$ of $\mathrm{MHB}$ and grown overnight at $37^{\circ} \mathrm{C}$. The bacteria suspension was then diluted to a 0.5 McFarland standard in $\mathrm{MHB}$ by measuring the turbidity at $625 \mathrm{~nm}$ yielding $1-2 \times 10^{8} \mathrm{CFU} / \mathrm{mL}$. A cotton swab was used to streak a MHA plate lawn. $4 \pm 0.5 \mathrm{~mm}$ deep MHA plates were created by adding $25 \mathrm{~mL}$ of molten MHA to a $9 \mathrm{~cm}$ round plate and left to solidify at room temperature. Tetracycline $(30 \mu \mathrm{g})$ and lincomycin $(15 \mu \mathrm{g})$ antibiotic disks (both Oxoid, UK) were applied to the MHA plates using a disk dispenser (Oxoid, UK), the plates were incubated for 18 hours at $35 \pm 1^{\circ} \mathrm{C}$ following EUCAST guidelines. Zone of inhibition diameters were illuminated using a lightbox and the images measured in $\mathrm{mm}$ using a ruler, the diameter was measured across 3 lines and the mean determined to be the zone of inhibition.

\section{Cell viability of $\mathrm{HaCaT}$ cells treated with free $A B X$ and Carbopol nanogel-formulated $A B X$.}

HaCaT cells (an immortalised human keratinocyte cell line, sourced from AddexBio, San Diego) were cultured in DMEM supplemented with $10 \% \mathrm{FBS}$ and $1 \%$ L-Glutamine in humidified conditions at $37^{\circ} \mathrm{C}$, $5 \% \mathrm{CO}_{2}$ in $\mathrm{T75}$ flasks until a confluency of $80 \%$ was achieved, determined by visualisation with a light microscope. Passaging was done at $80 \%$ confluency to ensure the cells remained in the exponential phase for experimentation. Passaging was performed by removing spent media and washing in DPBS and incubating with $1 \mathrm{x}$ Trypsin at $37{ }^{\circ} \mathrm{C} 5 \% \mathrm{CO}_{2}$ for 5 min until the cells were detached and in suspension. The trypsin was then neutralised with using 1:1 volumetric ratio of fresh DMEM and gently centrifuged at $1000 \mathrm{rpm}$ for $5 \mathrm{~min}$, the supernatant was aspirated, and the pellet resuspended in DMEM (supplemented as above) at a 1:6 ratio and transferred into a fresh T75 flask. Surplus cells were diluted and $1 \times 10^{4} \mathrm{HaCaT}$ cells were seeded in $50 \mathrm{~mL}$ of DMEM ( $A B X$ free) supplemented with 2 wt\% FBS (foetal bovine calf serum) into a 96-well plate and incubated for 24 hours at $37{ }^{\circ} \mathrm{C} 5 \% \mathrm{CO}_{2}$. The medium was then removed and replaced with treatment infused media (free $A B X$ or the formulated nanogel-encapsulated $A B X$ ) and incubated for 24 hours after which it was replaced with fresh DMEM and $20 \mu \mathrm{L}$ of CellTiter $96^{\circledR}$ Aqueous One Solution Reagent ${ }^{\mathrm{TM}}$ (Promega, UK) where it was incubated for 3 hours at the same conditions. The absorbance was then read at 492 $\mathrm{nm}$. These data were calculated into cell count data using the BeerLambert extinction coefficient law using absorbance values from a fixed number of cells in media. The HaCaT cell viability assay was repeated in three independent experiments.

\section{Minimum Inhibitory Concentration (MIC)}

The bacteriostatic properties of the bPEl-coated ABX-loaded Carbopol nanogel particles and its spectrum of activity were measured in comparison to equivalent concentrations of free tetracycline or lincomycin. The antimicrobial activity was tested against each of the strains described in Table 1 . Briefly, $\mathrm{O} / \mathrm{N}$ cultures of each strain were prepared as described in above. $100 \mu \mathrm{L}$ of each strain suspension was added to the wells of a 96-well microtiter plate yielding $5 \times 10^{4}-1 \times 10^{5}$ cell per well. An aliquot of bPEI-coated-0.2 wt $\%$ ABX-0.2 wt\% Carbopol suspension was created. This was centrifuged at $4000 \mathrm{rpm}$ for 5 minutes and re-suspended into $10 \mathrm{~mL}$ of MHB. 2-fold dilutions of this suspension were created in separate aliquots and $50 \mu \mathrm{L}$ added to descending rows of bacteria on the microtiter plate. Equivalent 2-fold dilutions of the free $A B X$ were added to separate columns to for comparison ( $50 \mu \mathrm{L}$ per well). The plates were incubated for 20 hours at $37{ }^{\circ} \mathrm{C}$. $20 \mu \mathrm{L}$ of resazurin sodium salt $(0.15 \mathrm{mg} / \mathrm{mL}$ in DPBS) was added to each well and incubated at $35{ }^{\circ} \mathrm{C}$ for 2 hours. The absorbance was recorded using a spectrophotometer (Jenway 7310, Cole-Parmer). The resazurin in the presence of viable cells is reduced to resorufin by the bacterial co-enzyme NAHD, indicating the cells remain metabolically active and viable (see Figure S1, ESI). The MIC was determined to be the lowest concentration of active antimicrobial agent which inhibited the growth of each strain. The MIC assay was repeated in three independent experiments.

\section{Time-kill assays}

Killing curves were performed against each representative strain to determine the time taken for the active agent in influence total bacterial cell populations. $\mathrm{O} / \mathrm{N}$ cultures were prepared yielding a 10 $\mathrm{mL} \mathrm{MHB}$ aliquots with $5 \times 10^{5}-1 \times 10^{6}$ cells per $\mathrm{mL}$. $10 \mathrm{~mL}$ of 0.005 wt\% bPEl-coated-0.2 wt\% ABX-0.2 wt\% Carbopol nanogel was added to each tube and incubated for 24 hours at $37{ }^{\circ} \mathrm{C}$ with $140 \mathrm{rpm}$ shaking. At time points $0,1,2,3,4,6$, and 24 hours a $100 \mu \mathrm{L}$ sample was removed from each treatment tube and added to $900 \mu \mathrm{L}$ of DeyEngley Neutralising broth. $100 \mathrm{~mL}$ aliquots from each serial dilution tube were plated onto MHA plates and incubated for 24 hours at 37 ${ }^{\circ} \mathrm{C}$ and enumerated. The time-kill assay was repeated in three independent experiments.

\section{Statistical analysis}

Data were expressed as average values \pm standard deviations of the mean. $P$-values of less than 0.05 were considered significant. All OneWay ANOVAs and Tukey's post-test statistical analysis were performed in GraphPad v7.0.4. 

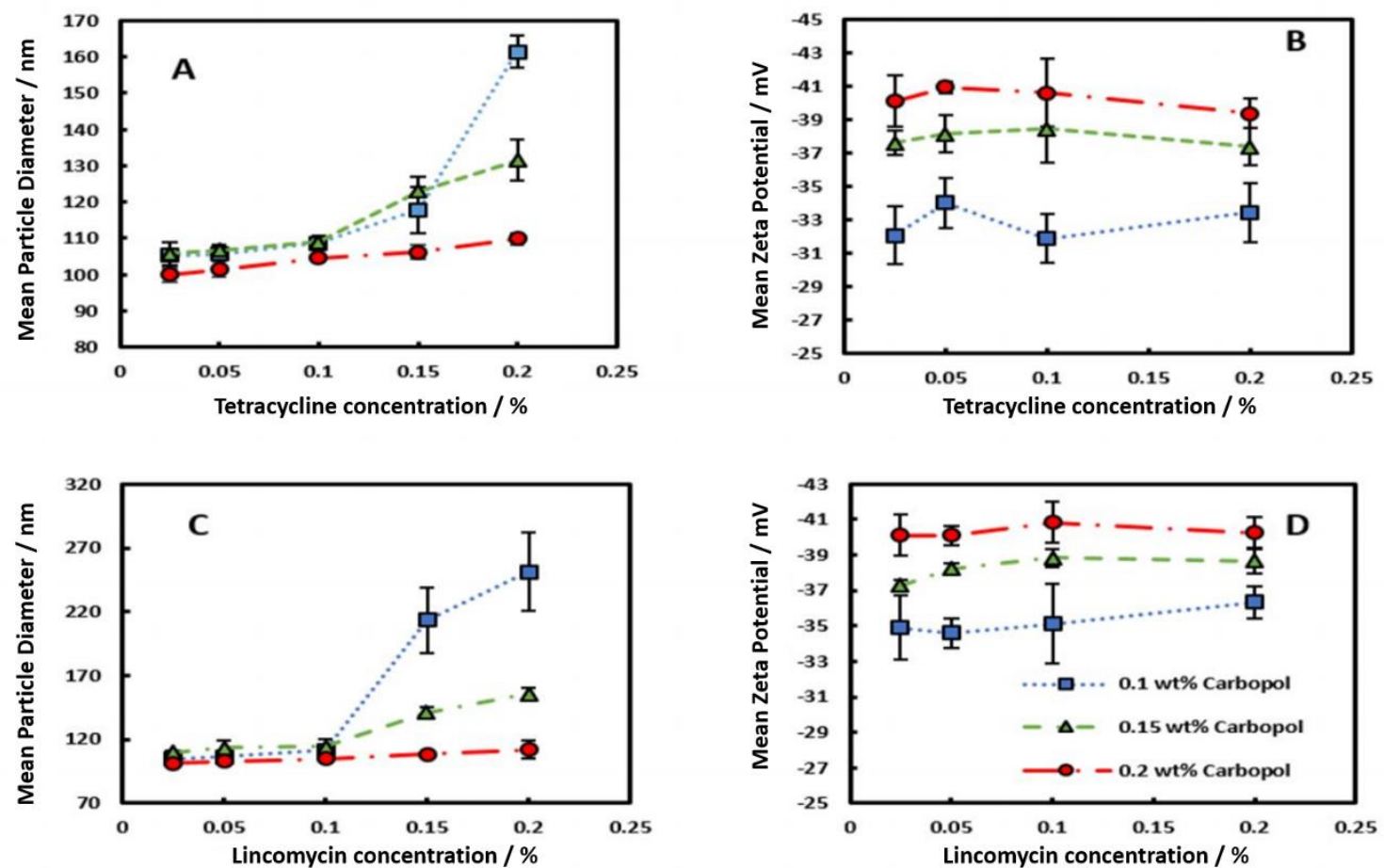

Figure 3. (A) The mean particle diameter and (B) zeta potential of $0.1,0.15$, and $0.2 \mathrm{wt} \%$ Carbopol Aqua SF1 nanogel after encapsulation of tetracycline at various concentrations and (C) The particle diameter and (D) zeta potential of 0.1, 0.15, and $0.2 \mathrm{wt} \%$ Carbopol Aqua SF1 nanogel after encapsulation of lincomycin at various concentrations at pH 6. 0.1, 0.15, and 0.2 wt\% Carbopol Aqua SF1 nanogel suspensions were created and mixed with tetracycline/lincomycin hydrochloride at varying concentrations. Each value represents a triple replicate with \pm S.D. The lines are guides to the eye. The legend is representative of all Figures $3 \mathrm{~A}, 3 \mathrm{~B}, 3 \mathrm{C}$, and $3 \mathrm{D}$.

\section{SEM imaging of the treated cells}

Cells were removed from their media by centrifugation at $2000 \mathrm{rpm}$ for $5 \mathrm{~min}$ and washed and resuspended in PBS buffer. The washing process was done three times to remove access media peptone. The cells were then fixed in a $1 \mathrm{wt} \%$ glutaraldehyde PBS buffer solution for 1 hour at room temperature, the cells were then centrifuged at $2000 \mathrm{rpm}$ and the pellet washed with deionized water three times to remove excess glutaraldehyde, the cell samples were then re-suspended in and dehydrated 50\%/75\%/90\% and absolute ethanol solutions for 30 minutes per each ethanol concentration. Cells were then swabbed onto a glass slide and submerged into absolute ethanol and dried using liquid $\mathrm{CO}_{2}$ at its critical point using an E3000 Critical Point Dryer (Quorum Technologies, UK) and then coated in $10 \mathrm{~nm}$ Carbon. The samples were imaged using an Ultra-High-Resolution Scanning Electron Microscope using cold field emission (Hitachi SU8230, Japan).

\section{Results and discussion}

Encapsulation of tetracycline and lincomycin into Carbopol Aqua SF1 nanogel
The particle size and zeta potential of Carbopol SF-1 were measured using a Malvern Zetasizer Nano ZS at $25^{\circ} \mathrm{C}$ using a refractive index of 1.450. A $30 \mathrm{wt} \%$ stock solution was diluted to 0.02 wt\% in deionized water. The Carbopol nanogel suspension has an average particle diameter of $100 \mathrm{~nm}$ and a zeta potential of $-30 \pm 1.82 \mathrm{mV}$ (see Figures $2 \mathrm{~A}$ and $2 \mathrm{~B}$, respectively). Figure $2 \mathrm{C}$ shows the useful swelling effect of the Carbopol Aqua SF1 nanogel and how pH changes in the solution can be used to mediate this. There is a change in the nanogel particle diameter from approximately $100 \mathrm{~nm}$ to $300 \mathrm{~nm}$ when adjusting the $\mathrm{pH}$ from 6 to 8 , which is due to the $\mathrm{COOH}$ groups of the Carbopol undergoing de-protonation to COO- in a basic solution. The electrostatic repulsion of these negatively charged -COO- groups causes the particle swelling effect and facilitates the subsequent electrostatic binding of cationic compounds. The negative zeta potential of the swollen nanogel, which remains between $-25 \mathrm{mV}$ and $-29 \mathrm{mV}$ at a $\mathrm{pH}$ range of 4 to 12 (Figure 2D), shows the anionic nature of the Carbopol SF1 nanogel. This was exploited to bind the cationic antibiotics ( $A B X)$, tetracycline hydrochloride, and lincomycin hydrochloride to the cores of the nanogel. The particle size of the de-swelled nanogel with encapsulated $A B X$ is dependent on the $A B X$ concentration with which the nanogel has been incubated. 


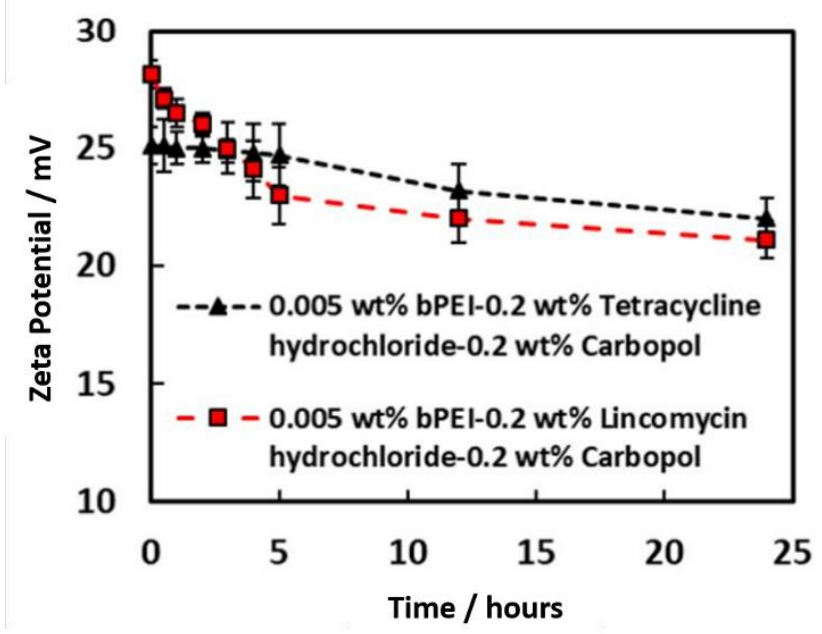

Figure 4. The zeta potential of bPEI coated antibiotic-Carbopol particles at $\mathrm{pH}$ 5.5 measured at time intervals after preparation. A Malvern Zetasizer was used to measure the particle diameter and zeta potentials at a refractive index of either 1.450 (below pH 7) or 1.336 (above pH 7). Each value represents average triplicate measurements with error bars representing the \pm S.D. The lines are guides to the eye.

Figures $3 A$ and $3 C$ shows the relationship between the $A B X$ concentration and the nanogel colloid stability which has to be maintained. This requires the $A B X$ concentration to be proportional to the nanogel concentration within certain range. For both the tetracycline and the lincomycin solutions loaded in the 0.2 wt\% Carbopol-0.2 wt\% ABX allows a nanogel particle shrink to approximately $110 \pm 2 \mathrm{~nm}$. However, for increased $A B X$ concentrations and lower concentrations of the nanogel, the particle may undergo aggregation and the formation of less stable nanogel, approximately $160 \pm 5 \mathrm{~nm}$ and $220 \pm 5.12 \mathrm{~nm}$ for tetracycline and lincomycin, respectively.

The optimal combination of 0.2 wt\% ABX-0.2 wt\% Carbopol Aqua SF1 nanogel formulation was chosen as this allowed the highest encapsulation efficiency of $A B X$ without compromising the nanocarrier colloidal stability. The zeta potential of the collapsed ABX-loaded nanogel was unaffected by the concentration of the encapsulated $A B X$. Figure $3 B$ and $3 D$ (tetracycline and lincomycin respectively) show the zeta potential of $0.2 \mathrm{wt} \%$ Carbopol remains stable at approximately $-39 \pm 1 \mathrm{mV}$ whether loaded with lower or higher concentrations of $A B X$, indicating that the $A B X$ concentration does not impact on the overall surface charge of the collapsed nanogel particles. The purpose of encapsulating the antibiotic was to provide a targeted delivery system which would release it in close proximity to the microbial cell wall. To achieve this the $0.2 \mathrm{wt} \%$ 0.2 wt\% $A B X$ formations were mixed with very low concentrations $(0.005 \mathrm{wt} \%)$ of the cationic polyelectrolyte bPEI (branched polyethyleneimine) to reverse the surface charge of the collapsed nanogel particles and induce electrostatic attraction to the negative bacterial cell wall. $0.005 \mathrm{wt} \% \mathrm{bPEI}$ was chosen as it was the optimal concentration that can reverse the surface charge of the nanogel particles and maintain optimal colloidal stability of the nanocarrier.
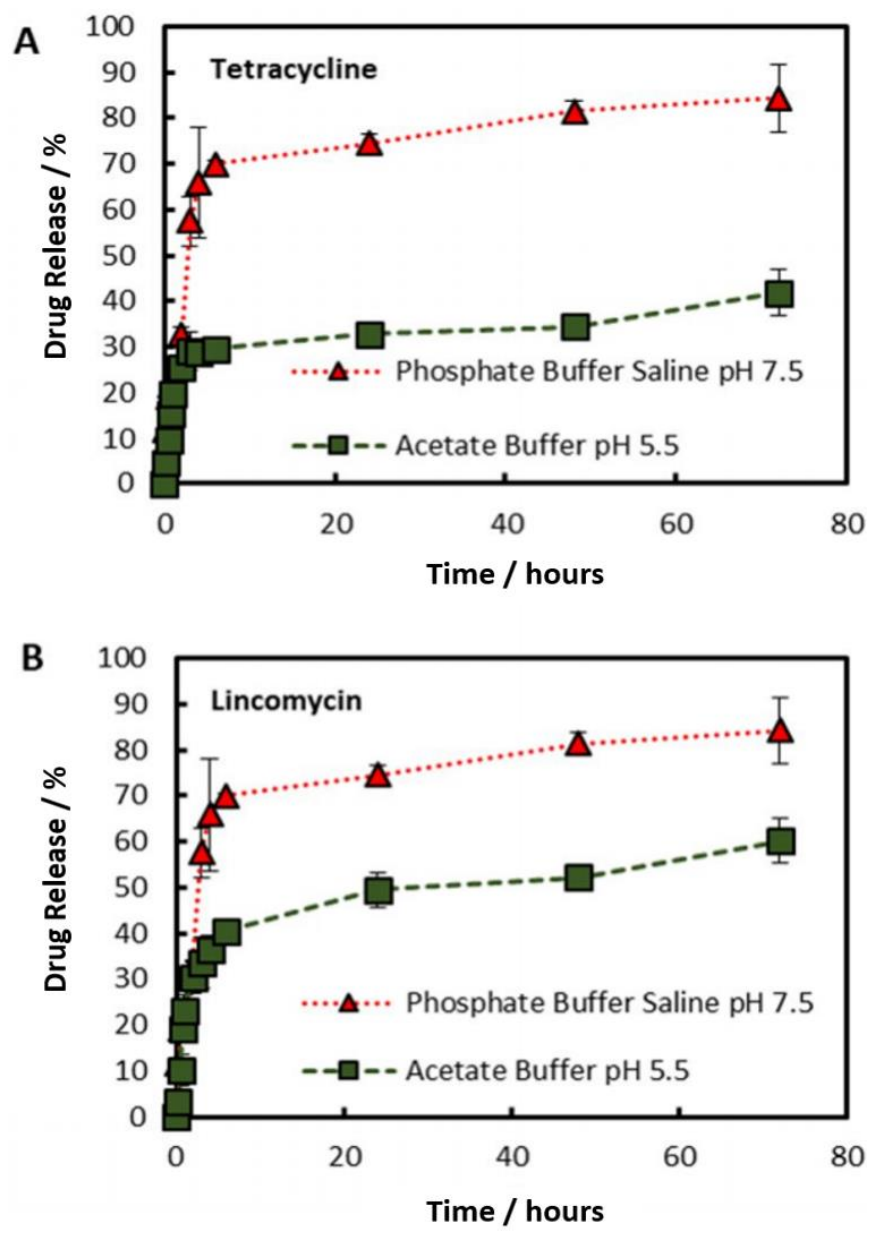

Figure 5. (A) Tetracycline and (B) lincomycin release kinetics in different $\mathrm{pH}$ solutions. $10 \mathrm{~mL}$ of $0.2 \mathrm{wt} \%$ ABX $-0.2 \mathrm{wt} \%$ Carbopol suspension was placed in a 10-12 kD MWKO dialysis bag. The dialysis bag was submerged into either $250 \mathrm{~mL}$ of phosphate or acetate buffer ( $\mathrm{pH} 7.5$ and 5.5, respectively) whilst stirred. $1 \mathrm{~mL}$ aliquots were taken at specific time points on the absorbance of the aliquot equated into the concentration of $A B X$ in the buffer using a standard calibration curve. Absorption was measured at $625 \mathrm{~nm}$.

Figure S3 (ESI) shows increased concentrations of bPEI-induced aggregation of the nanogel particles with no detectable further increase in their zeta potential. Figure 4 shows the zeta potential of the $A B X$-loaded nanogel particles was reversed from approximately $-39 \mathrm{mV}$ to $+(25-28) \mathrm{mV}$ for both tetracycline and lincomycin loaded in Carbopol Aqua SF1 after coating with bPEI. Our measurements showed that the zeta potential of the bPEI-coated nanogel particles remained very stable and positive for more than 24 hours indicating the bPEI has coated the particle surface, conferring a long-lasting positive surface charge.

\section{Encapsulation efficiency and release kinetics of tetracycline and lincomycin in Carbopol Aqua SF1}

The supernatant of the $A B X$-nanogel formation was retained after centrifuging to determine how much $A B X$ the nanogel has been encapsulated in the swelling and de-swelling process. Figure S2A and S2B (ESI) show the absorbance of the tetracycline and lincomycin in deionized water reduced to $\mathrm{pH} 5$ using $0.25 \mathrm{M} \mathrm{HCl}$. 


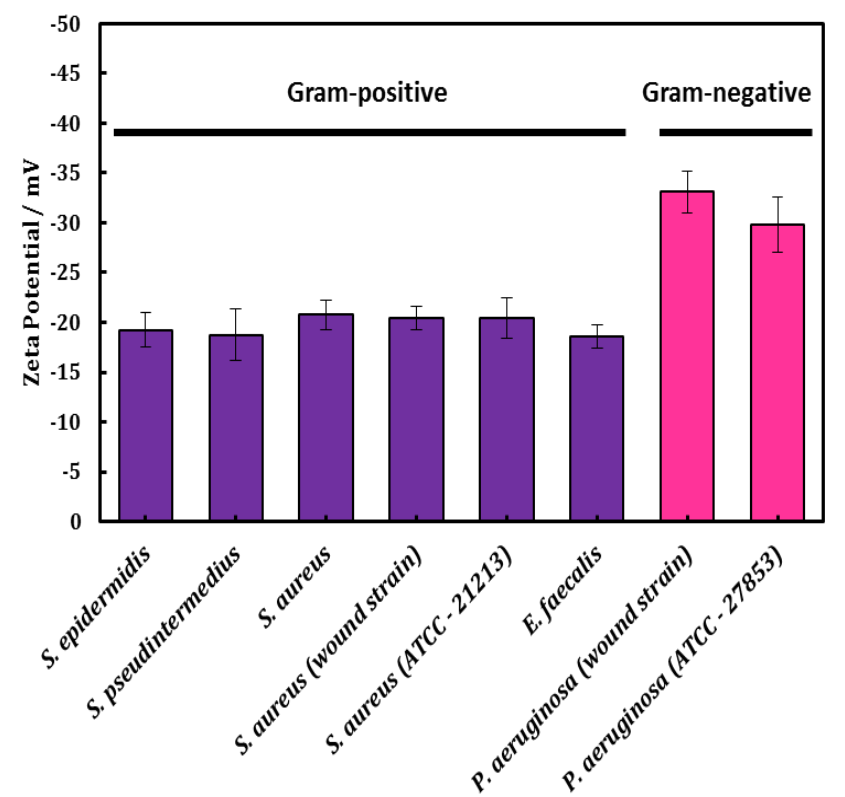

Figure 6. Zeta potential of bacterial wound species. A colony of each species was inoculated into $10 \mathrm{ml}$ of $\mathrm{MHB}$ and grown overnight at $37^{\circ} \mathrm{C}$ with constant shaking. The bacterial suspension was the centrifuged for $10 \mathrm{~min}$ at $5000 \mathrm{rpm}$. The supernatant was discarded, and the pellet washed twice with deionized water. The pellet was resuspended in $45 \mathrm{ml}$ of deionized water. $1 \mathrm{~mL}$ of this suspension was placed in a quartz cuvette and zeta potential was measured using the Dipstick probe and Malvern Zetasizer nano ZS. The refractive index was 1.384 and the absorption $1.000 . \mathrm{N}=3$ with \pm S.D.

This calibration curve was used to compare the absorbance readings obtained from the supernatant created during the centrifugal process used in forming the ABX-loaded nanogel particles. A linear regression equation was used to determine the $A B X$ concentration in the supernatant and from this the $A B X$ concentration retained within the nanogel. Tables S1 and S2 (ESI) show that increasing the temperature to $37^{\circ} \mathrm{C}$ while incubating the Carbopol nanogel and the $A B X$ solutions prior to mixing, and the duration of the mixing both affect the $A B X$ amount successfully encapsulated. In both the tetracycline and lincomycin formulations, the $30 \mathrm{~min} / 37^{\circ} \mathrm{C}$ mixing conditions yielded the highest encapsulation efficiency, with $89.9 \%$ for tetracycline and $79.5 \%$ for lincomycin, respectively. The release kinetics of $A B X$ from the $A B X$-loaded nanogel formations was tested to examine how the $A B X$ release is affected by the $\mathrm{pH}$ of the solution. Figures $5 \mathrm{~A}$ and $5 \mathrm{~B}$ show that tetracycline and lincomycin are slowly released from the interior of the nanoparticles over a period of 24 hours. In both the tetracycline and lincomycin loaded nanogelformulations, their release from the nanogel is higher in the PBS $\mathrm{pH}$ 7.5) compared to the acetate buffer ( $\mathrm{pH}$ 5.5). In both the acetate and phosphate saline buffers, there is a large release of both tetracycline and lincomycin within the first 5 hours between $30 \%$ to $70 \%$ for the acetate buffer and PBS, respectively. Further release of the antibiotic is much slower and gradual for the remaining time. The increased $A B X$ release rates in the phosphate buffer solutions can be explained by the partial swelling of the nanogel at the higher $\mathrm{pH}$, which will allow $A B X$ to diffuse faster from its interior.
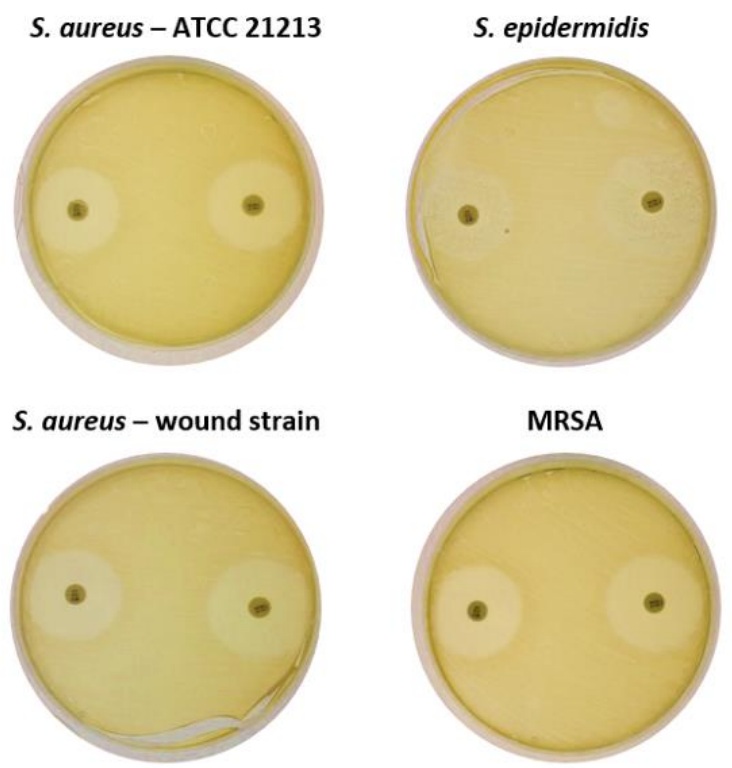

E. faecalis
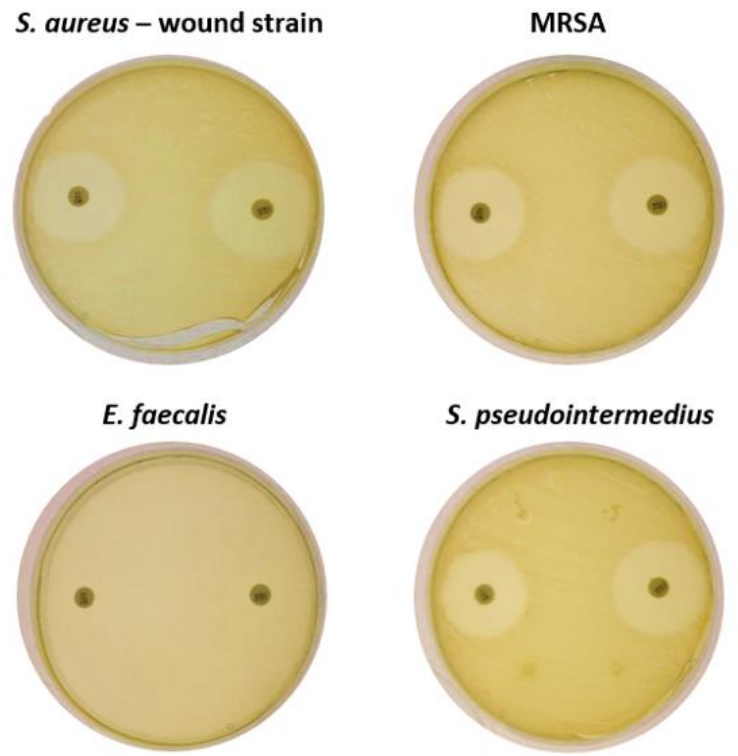

S. pseudointermedius

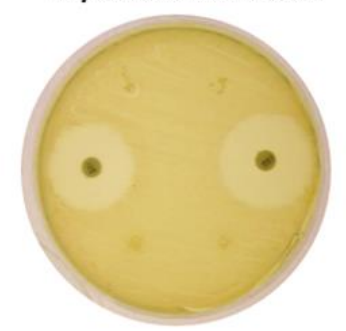

P. aeruginosa - wound strain
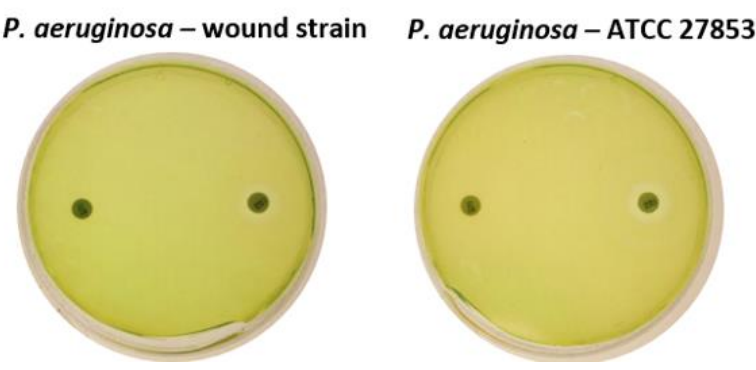

Figure 7. Zones of inhibition (AST disk diffusion test) for wound isolates and reference strains. $15 \mu \mathrm{g}$ lincomycin disks (left) and $30 \mu \mathrm{g}$ tetracycline disks (right).

The continued release of $A B X$ from the nanogel particles over a long period of time could also potentially be highly beneficial in an antimicrobial context, as the ABX will be delivered locally on the surface of the bacterial cell continuously for a long period of time.

We are not aware of previous studies in which these antibiotics were encapsulated into nanogel particles of the same nanocarrier chemistry. These results allow comparison with typical encapsulation efficiencies with other cationic antimicrobial molecules such as berberine hydrochloride ${ }^{31}$ and chlorhexidine digluconate. ${ }^{32,23}$ Our studies show that encapsulation of these cationic antimicrobials has proven successful within anionic microgels and dual functionalised shellac nanocarriers with comparable encapsulation efficiency. 


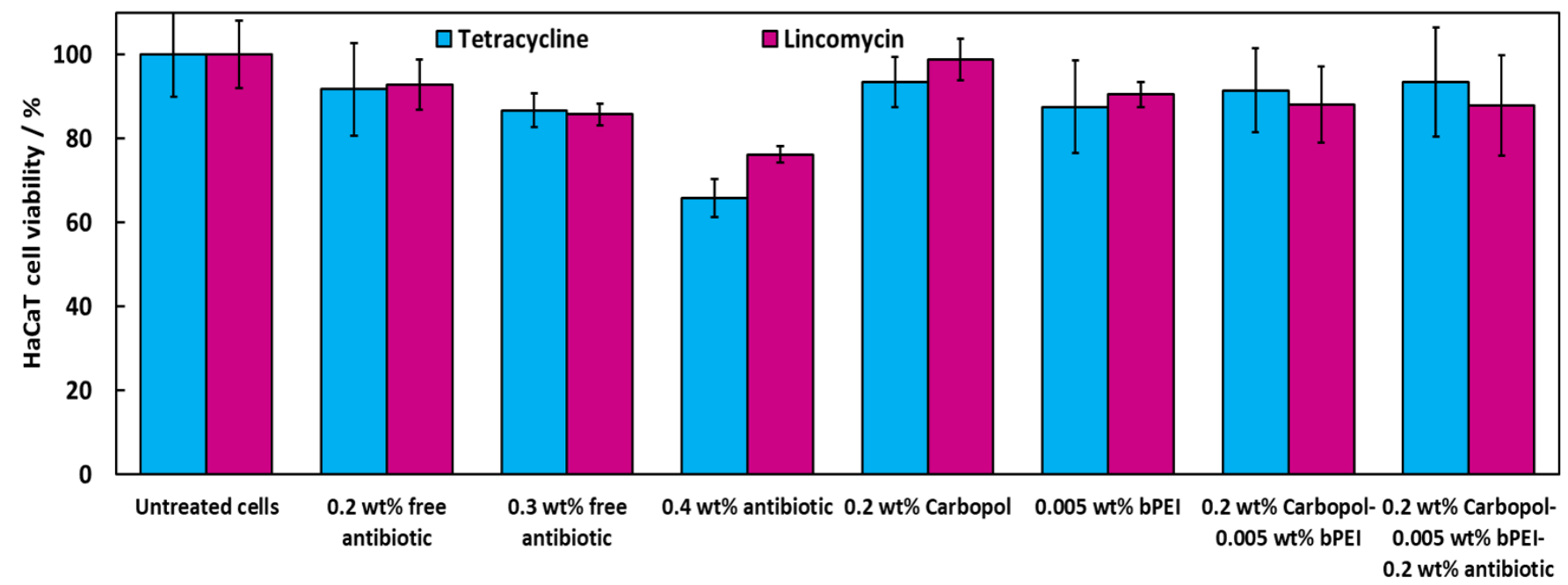

Figure 8. HaCaT viability after treatment with tetracycline concentrations and Carbopol formulated tetracycline/lincomycin and individual formulation constituents. $1 \times 10^{4} \mathrm{HaCat}$ cells were seeded in $50 \mathrm{ml}$ of DMEM (antibiotic free) supplemented with $2 \mathrm{wt} \%$ FBS (foetal bovine calf serum) into a $96-$ well plate and incubated for 24 hours at $37{ }^{\circ} \mathrm{C}$ in $5 \% \mathrm{CO}_{2}$ atmosphere. The medium was then removed and replaced with treatment infused media.

Figure S4 (ESI) shows SEM images acquired of (A) a sample of 0.2 wt\% Carbopol loaded with 0.2 wt\% lincomycin, (B) 0.005 wt\% bPEI-0.2 wt\% lincomycin-0.2 wt\% Carbopol, and (C) 0.2 wt\% Carbopol nanogel (empty nanocarrier). Lincomycin was chosen for the characterisation due to the presence of a sulphur in the molecular structure. One can see from the EDX spectra the presence of sulphur in the lincomycin-loaded Carbopol nanogel particles after the particles have been washed from the excess of lincomycin. This spectra peak is not present in the unloaded nanocarrier (Figure S4C). We conclude from this result that lincomycin has been successfully loaded into the nanogel particles, also in agreement with the dynamic light scattering results presented in Figure 5.

\section{Zeta potential of wound-relevant bacterial species}

The action of the Carbopol encapsulated ABX, functionalised with bPEI to yield a positive surface charge is to facilitate a sustained and targeted $A B X$ delivery platform, which is based on the electrostatic adhesion between the nanogel particles and the bacterial cell wall. Seven wound-associated bacterial pathogens, along with a $S$. aureus reference strain (ATCC - 21213) and $P$. aeruginosa reference strain (ATCC - 27853) were cultured overnight and centrifuged for 10 minutes at $5000 \mathrm{rpm}$. The pellet was re-dispersed into deionized water for zeta potential measurement. The zeta potential for Gramnegative species was between $-30 \mathrm{mV}$ and $-37 \mathrm{mV}$, while the six Gram-positive species had a very similar zeta potential of $-20 \pm 2 \mathrm{mV}$ (Figure 6). As expected, the additional negatively charged lipopolysaccharide (LPS) confers higher negative surface charge for the Gram-negative species. Crucially, all bacteria tested displayed a negative charge which should allow electrostatic adhesion between the bacterial cell wall and the cationic nanogel formulations.

\section{Antimicrobial susceptibility testing (AST) Disk Diffusion Test of wound species}

Tetracycline and lincomycin disks were placed on bacterial lawns of the nine selected bacterial species to confirm antibiotic resistance (Figure 7). The zone of inhibition surrounding the disk was measured in $\mathrm{mm}$ using a ruler and compared to reference values from the European committee of antimicrobial susceptibility testing (EUCAST). ${ }^{34}$ Table S3 (ESI) shows the results from the antimicrobial susceptibility disk diffusion testing. S. aureus (both strains), $S$. epidermidis, MRSA, and S. pseudointermedius were susceptible to both tetracycline and lincomycin. $P$. aeruginosa (both strains) and $E$. faecalis were resistant to both tetracycline and lincomycin. Figure 7 shows the clear zones of inhibition which surround the species which confer resistance to either antibiotic.

\section{Viability of $\mathrm{HaCaT}$ cells treated with tetracycline/lincomycin and Carbopol encapsulated tetracycline/lincomycin and its individual constituents}

A preliminary human cell cytotoxicity experiment was performed on $\mathrm{HaCaT}$ cells to assess if the Carbopol Aqua SF1 nanogel formulations (and individual constituents) were toxic to human cells compared to the free antibiotics. Interestingly, Figure 8 shows that after 24 hours of incubation $0.2 \mathrm{wt} \%, 0.005 \mathrm{wt} \% \mathrm{bPEI}$, and $0.2 \mathrm{wt} \% \mathrm{bPEI}-$ $0.2 \mathrm{wt} \%$ Carbopol (empty carrier) showed no toxic effect compared to the untreated cell controls. This indicates the individual components of the nanogel formulation did not lead to a reduction in viable cell number after 24 hours. Treatments with free tetracycline and lincomycin were used at concentrations $0.2,0.3$, and $0.4 \mathrm{wt} \%$ and showed a slight reduction in total cell counts when compared to the control, approximately $20 \%$ to $25 \%$ for lincomycin and tetracycline at 0.4 wt\% respectively. The 0.2 wt\% Carbopol-0.2 wt\% ABX-0.005 wt\% bPEI formulation showed toxicity in line with $0.2 \%$ free antibiotic. This indicates that the nanogel-loaded antibiotics confer no increased toxicity to $\mathrm{HaCaT}$ cells, both when 
encapsulating an antibiotic and as an empty nanocarrier. This formulation could have a potential topical application to treat infected wounds. HaCaT cells represent a good proxy for a typical skin keratinocyte which would be exposed to the ABX-loaded nanocarrier formulation during the wound treatment.
S. aureus (ATCC - 21213)

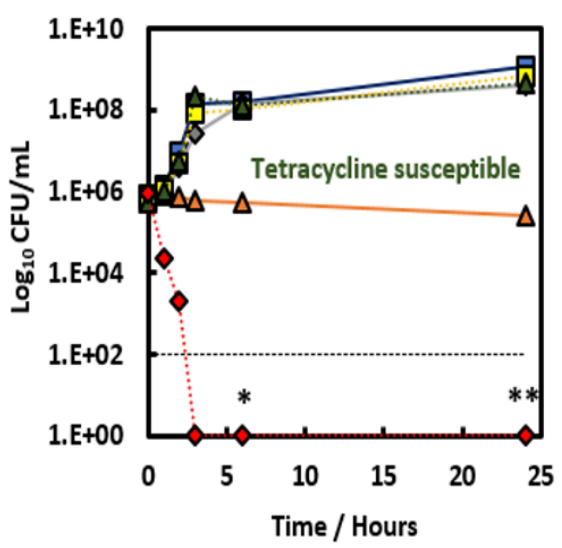

MRSA

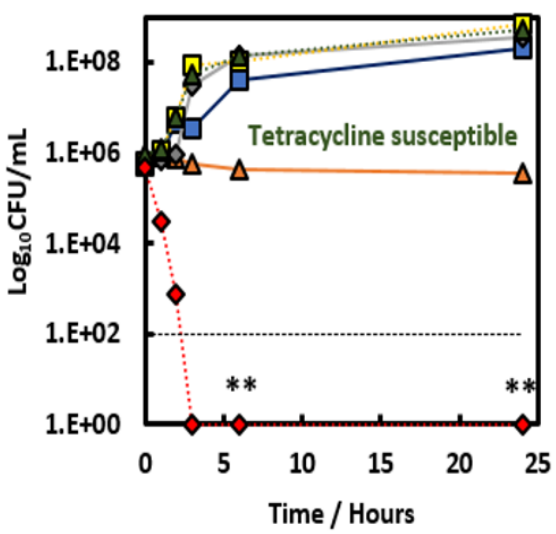

P. aeruginosa (wound strain)

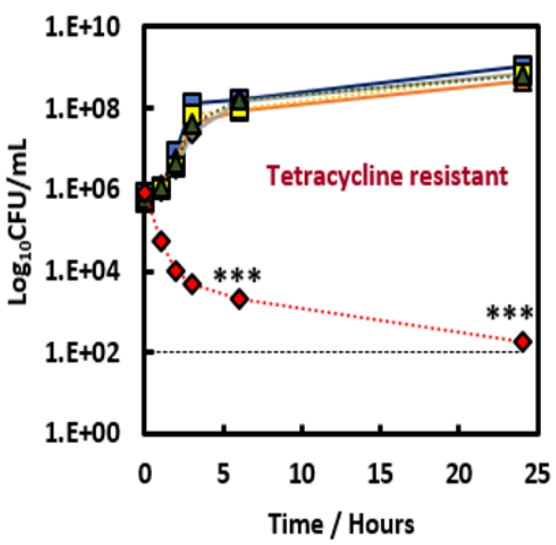

S. aureus (wound strain)

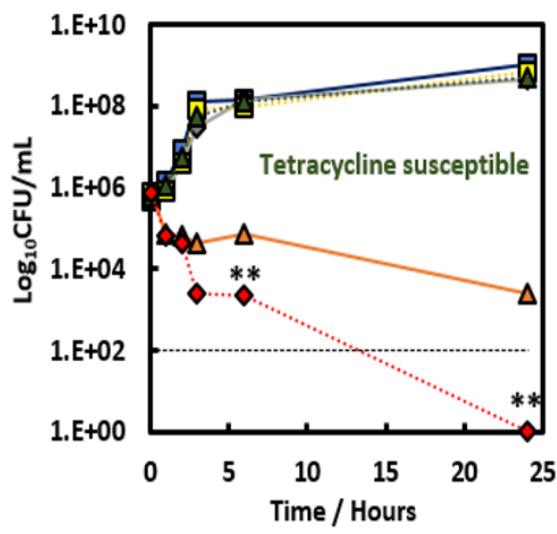

S. pseudointermedius

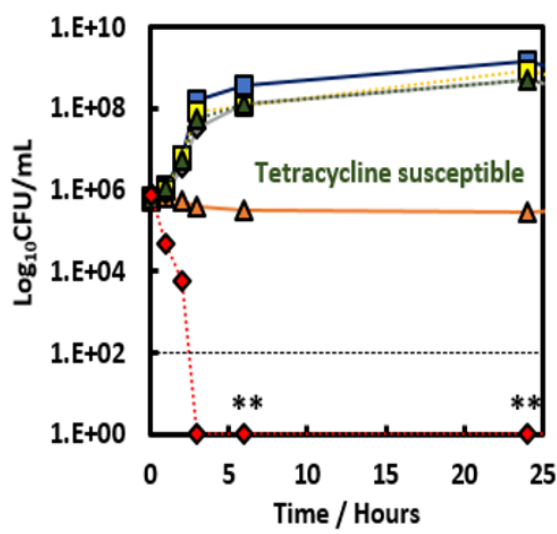

\section{E. faecalis}

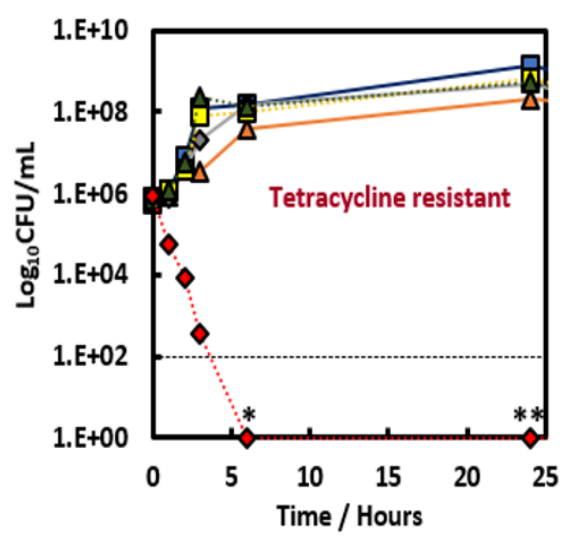

S. epidermidis

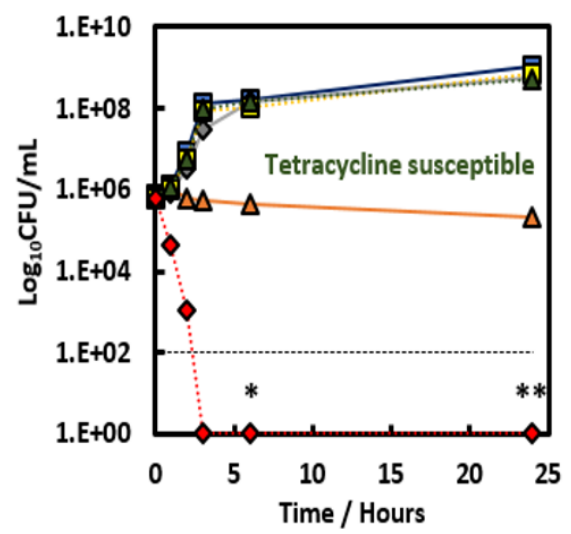

P. aeruginosa (ATCC - 27853)

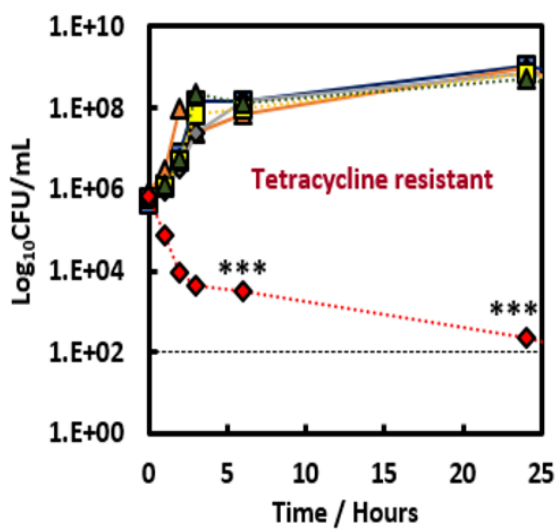

$\rightarrow-$ Growth control

$\Delta-0.2 w t \%$ tetracycline

$\diamond 0.005 w t \%$ bPEI

․ $0.2 w t \%$ Carbopol

....... 0.005 wt\% bPEI-0.2 wt\% Carbopol

..... 0.005 wt\% bPEI-0.2 wt\% Carbopol$0.2 \mathrm{wt} \%$ tetracycline Limit of detectability

Figure 9. Time kill assays against clinical wound isolates and selected reference species. Tetracycline and 0.005 wt $\%$ bPEl-coated 0.2 wt $\%$ tetracycline loaded in 0.2 wt\% Carbopol (CLC), $0.005 \mathrm{wt} \%$ free bPEI and $0.005 \mathrm{wt} \% \mathrm{bPEI}$-coated $0.2 \%$ Carbopol (empty nanocarrier) tested against several clinical isolate wound bacteria. The results are presented as means $(n=3)$. The lines are guides to the eye. $<0.05$ is considered significant. ${ }^{*} P<0.05,{ }^{* *} P<0.01,{ }^{* * *} P<0.001$ compared to the free tetracycline. 
S. aureus (ATCC - 21213)

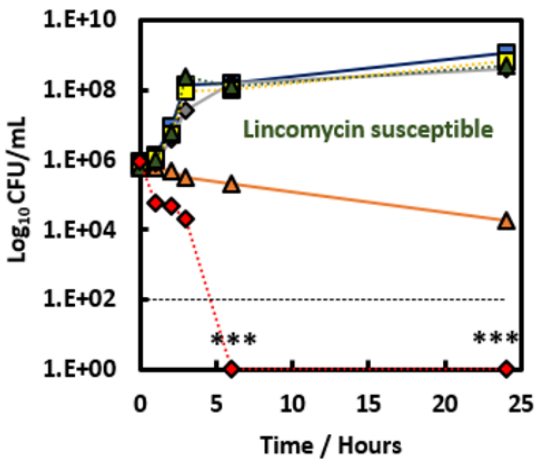

MRSA

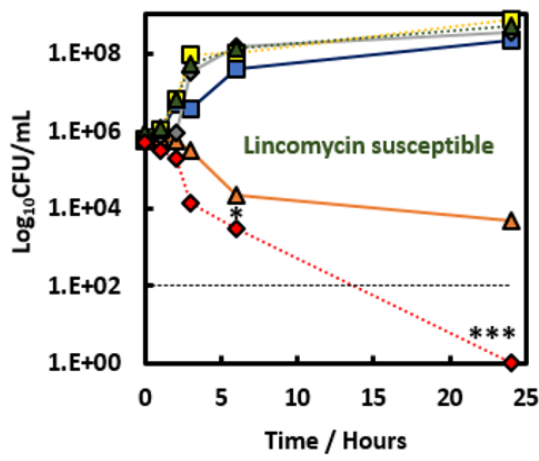

P. aeruginosa (wound strain)

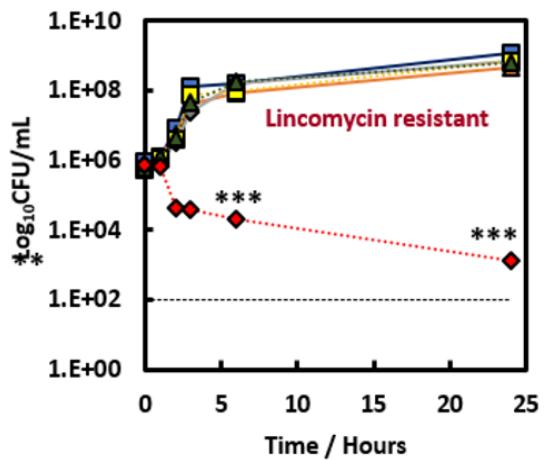

S. aureus (wound strain)

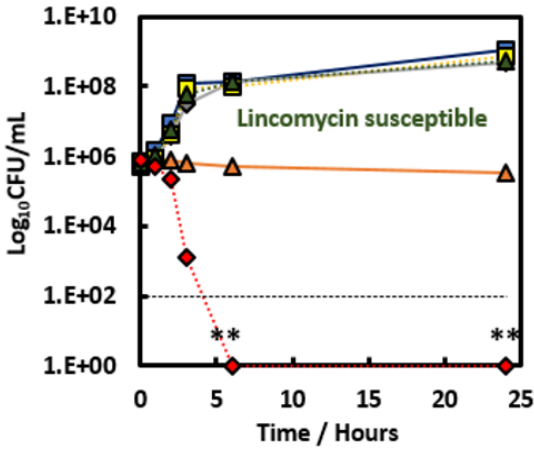

S. pseudointermedius

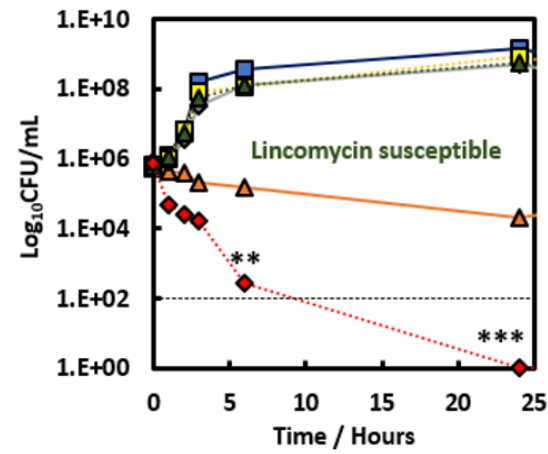

E. faecalis

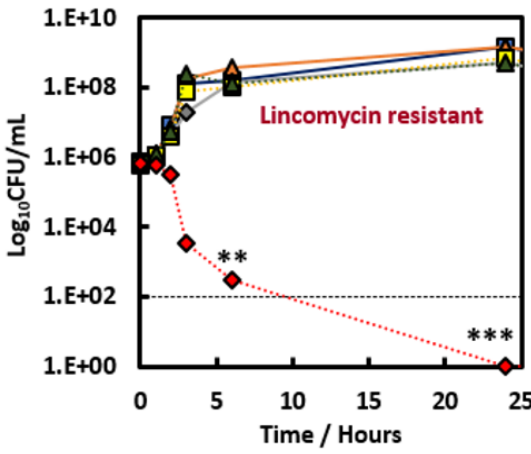

S. epidermidis

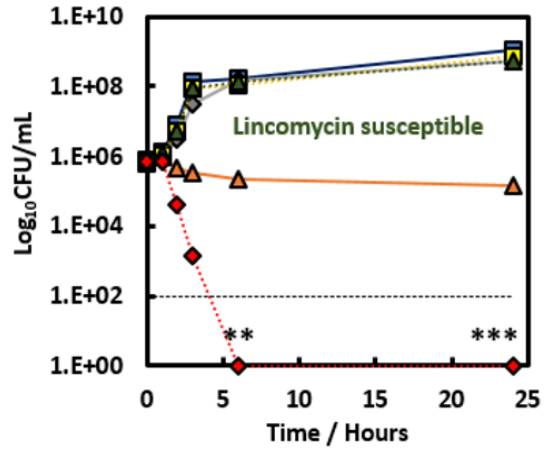

P. aeruginosa (ATCC - 27853)

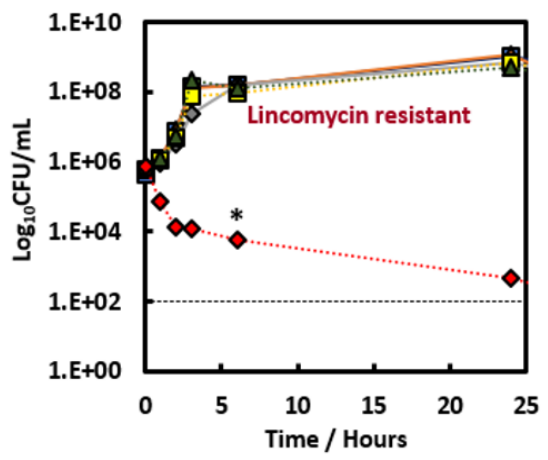

$\square-$ Growth control

$\triangle-0.2$ wt\% lincomycin

$\diamond-0.005$ wt\% bPE

ㅁ. 0.2 wt\% Carbopol

$\cdots \cdots . .005$ wt $\%$ bPEl-0.2 wt\% Carbopol

$\checkmark . . .0 .005$ wt\% bPEI-0.2 wt\% Carbopol$0.2 \mathrm{wt} \%$ lincomycin

Limit of detectability

Figure 10. Time kill assays against clinical wound isolates and selected reference species. Lincomycin and 0.005 wt\% bPEl-coated 0.2 wt\% lincomycin loaded in 0.2 wt\% Carbopol (CLC), 0.005 wt\% free bPEI and 0.005 wt\% bPEI-coated $0.2 \%$ Carbopol (empty nanocarrier) tested against several clinical isolate wound bacteria. The results are presented as means $(\mathrm{n}=3)$. The lines are guides to the eye. $<0.05$ is considered significant. $* \mathrm{p}<0.05, * * \mathrm{p}<0.01, * * * \mathrm{p}<0.001$ compared to free lincomycin.

Antibacterial activity of bPEI-coated tetracycline/lincomycin loaded Carbopol Aqua SF1 on clinical wound isolates

Figures 9 and 10 shows time kill evaluation of 0.005 wt\% bPEI0.2 wt\% Carbopol-0.2 wt\% ABX (tetracycline and lincomycin respectively). The uncoated Carbopol nanogel and bPEI coated nanogel were included as unloaded controls. Exponential growth was observed over 24 hours for the nine woundrelevant bacteria under no treatment conditions and in the presence of each control component $(0.2 \mathrm{wt} \%$ nanogel, 0.005 wt\% bPEI, and the 0.005 wt\% coated 0.2 wt\% nanogel). $S$. aureus (clinical isolate and reference strain), S. epidermidis, MRSA, and S. psuedointermeduis showed after 24 hours that both tetracycline and lincomycin had a bacteristatic effect on growth, with a small reduction in bacteria number over the 24 hours. By contrast, $P$. aeruginosa (clinical isolate and reference strain) and $E$. faecalis showed no reduction in cell population when treated with 0.2 wt\% free tetracycline and lincomycin compared to the untreated growth control. These results are in agreement with the AST disk diffusion results shown in Figure 7. 

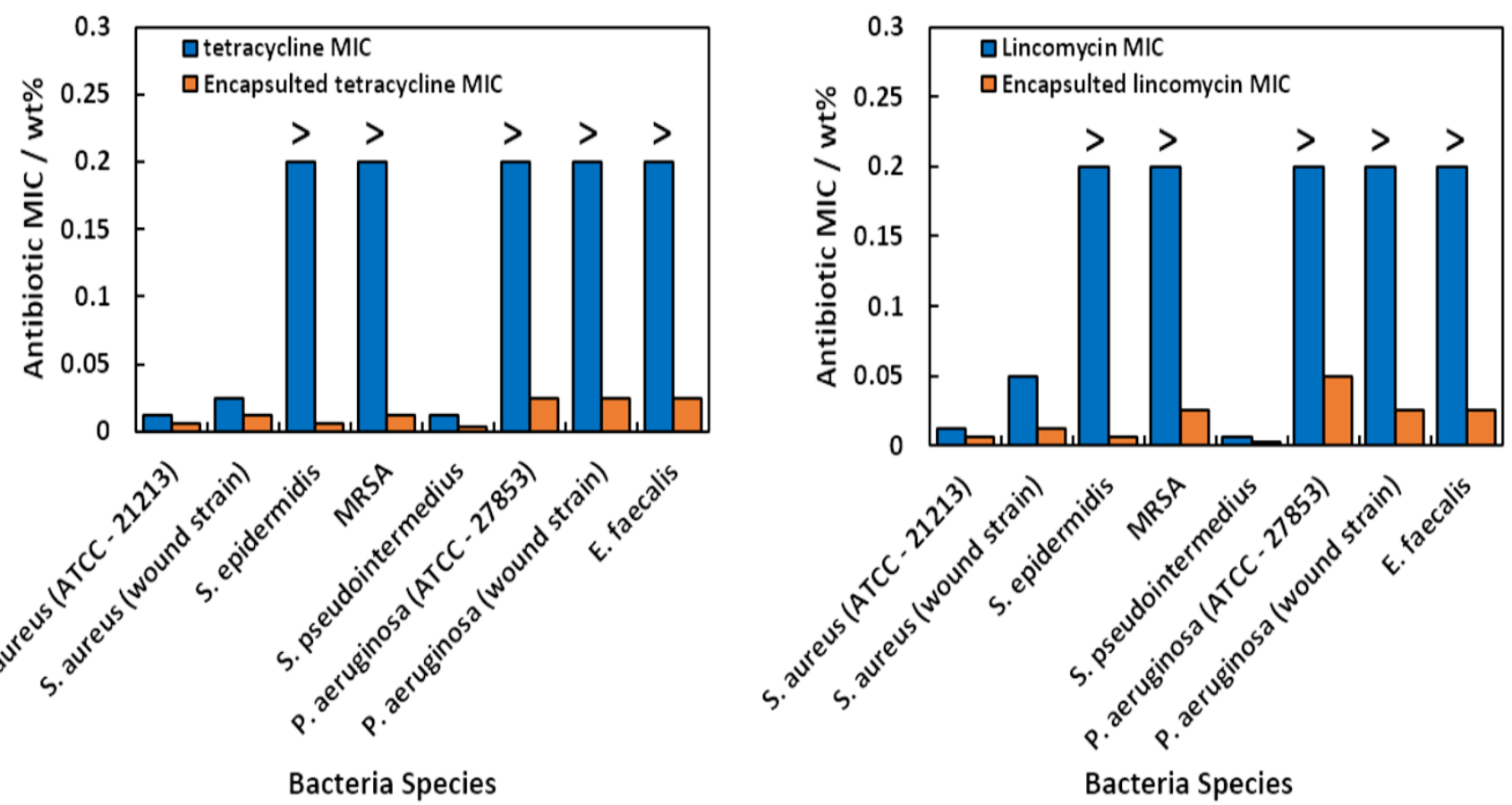

Figure 11. (A) MIC of tetracycline and nanogel-encapsulated tetracycline, (B) MIC of lincomycin and nanogel-encapsulated lincomycin. All nanogel particles were coated with bPEI. " $>$ " indicates that a concentration greater than $0.2 \mathrm{wt} \%$ is needed to inhibit bacterial growth.

The bPEl-coated nanogel encapsulated tetracycline and lincomycin were highly effective against $S$. aureus (reference strain) and $S$. epidermidis causing a $>5 \log$ reduction in cell population after approximately 3 hours. A similar result is observed in MRSA, $S$. psuedointermedius and $E$. faecalis with a $>5$ log reduction observed with the nanogel-encapsulated tetracycline at 3 hours, and after 24 hours with lincomycin. After 24 hours the wound isolated S. aureus has shown a $>5$ log reduction with encapsulated tetracycline and a $>1$ log reduction with encapsulated lincomycin. P. aeruginosa (ATCC and clinical isolate) showed an appromimate $>4$ log reduction with the nanogel-encapsulated tetracycline and lincomycin compared to equivalent concentrations of the free antibiotic. Collectively, these data show that the cationic antibiotic delivery system enhances antimicrobial action of tetracycline and lincomycin, across all nine bacteria evaluated in this study. Crucially, cationic nanocarrierbased antibiotic delivery was able to overcome antimicrobial resistance across several isolates, conferring $>5$ log reduction within 24 hours (Table S6, ESI).

The minimum inhibitory concentrations (MIC) for tetracycline and lincomycin are shown in Figure 11, the data is also shown in Tables S4 and S5 (ESI). The ability of the nanogel-encapsulated tetracycline to inhibit growth was demonstrated through all nine species with up to $8 \times$ MIC reductions shown. The $P$. aeruginosa strains also showed much reduced MIC concentrations when compared to free tetracycline, both $8 \times$ MIC. This effect was also mirrored with the nanogel-encapsulated lincomycin when compared to free lincomycin. Similar to nanogel-encapsulated tetracycline, there were reductions in the MIC of nanogel-encapsulated lincomycin compared to free lincomycin in solution. This was seen across all eight species, ranging from $8 \times$ MIC to $2 \times$ MIC. Interestingly, in the $P$. aeruginosa (ATCC strain and clinical isolate) and $E$. faecalis there was $8 \times$ MIC compared to free lincomycin, providing evidence the nanogel-encapsulated lincomycin also facilitates an improved inhibitory effect. The increased antimicrobial efficiency of the nanogel-encapsulated antibiotics would also be advantageous in case of susceptible bacteria as a reduced concentration of antibiotic is required to achieve a comparable result to that of the free antibiotic. This has potential benefits in reducing possible toxicities associated with antibiotic use, and a potential reduction in cost, as the antibiotic is considerably more expensive than the Carbopol nanogel Aqua SF1 and bPEI.

The most important benefit of the nanogel-encapsulated tetracycline and lincomycin formulations is their ability to be effective against species which have demonstrated resistance to these antibiotics. We envisage that this could be due to the sustained high concentration of the antibiotic localised to the cell wall of the bacteria being able to overwhelm efflux pumps, which are able to actively remove free antibiotic. Figure 12 shows SEM images of P.aeroginosa after treatment with bPEI-coated tetracycline loaded Caropol nanogel which demonstrates the accumulation of the surface functionalised nanogel particles on the surface of the anionic bacterial cell wall (described and illustrated above). 

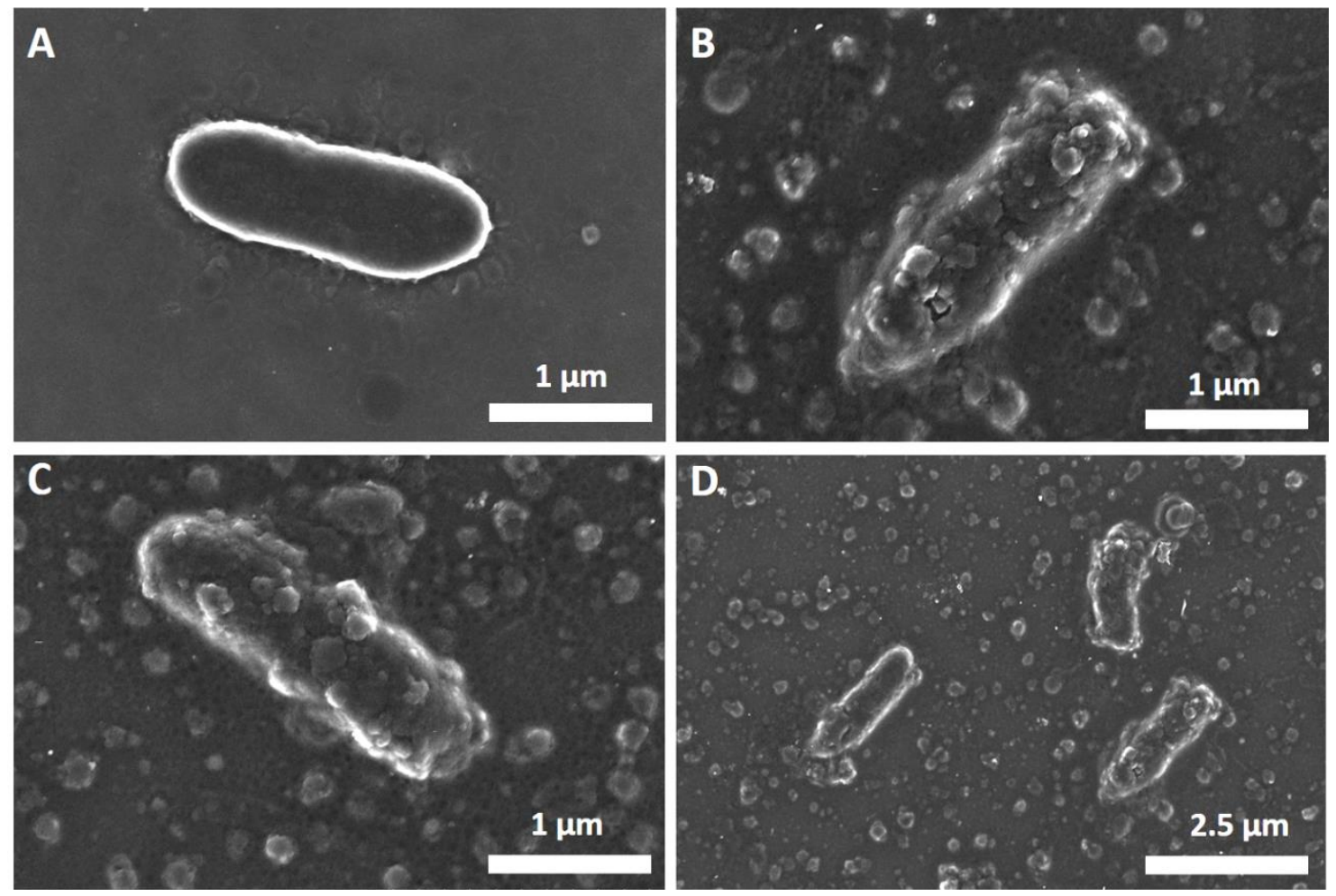

Figure 12. SEM images of $P$. aeruginosa before and after treatment. (A) shows the control sample of non-treated $P$. aeruginosa after 24 hours of growth. (BD) shows $P$. aeruginosa after 24 hours treatment with 0.005 wt $\%$ bPEI- 0.2 wt\% tetracycline- 0.2 wt $\%$ Carbopol.

\section{Conclusions}

We have developed a novel surface functionalised nanocarrier for tetracycline and lincomycin by using lightly cross-linked polyacrylic acid-based nanogels (Carbopol Aqua SF1) coated with a biocompatible cationic polyelectrolyte (bPEI). We show that this approach significantly enhanced the antimicrobial action against a range of wound isolated pathogens. Crucially, the bPEI surface coated nanogel with encapsulated antibiotics had an enhanced effect on several bacterial species which were validated to be resistant to either tetracycline or lincomycin. This enhanced antibiotic efficacy is likely due to favourable electrostatic attraction between the positively charged bPEl-coated antibiotic-loaded nanogel particles and the negatively charged bacterial cell wall. This is mediated by the attachment of the nanocarrier particles which then locally release the encapsulated antibiotic in much higher local concentrations than the free antibiotic at the same overall concentration. We also showed that the cytotoxicity of the antibiotics loaded in these nanocarriers on human keratinocyte cell is practically negligible at the range of concentrations which kill both resistant and susceptible bacteria. Antimicrobial resistance is a growing clinical epidemic. This novel method for bacteria-targeted topical antibiotic application offers an important new opportunity to extend the clinically useful life-span of existing antibiotics, using inexpensive materials, while reducing future bacterial antibiotic resistance.

\section{Supplementary Information}

The enclosed Electronic supplementary information file (ESI) contains tabulated data for the minimal inhibitory concentration for the free and nanocarrier-loaded se antibiotics on planktonic bacteria. We also enclose EDX and DLS data on the nanocarrier loaded with $A B X$ in comparison with the empty nanocarrier.

\section{Acknowledgements}

P.J.W. thanks the University of Hull for the PhD studentship as part of its Advanced Wound Care PhD cluster. All experiments performed by P.J.W. The authors would like to thank SEM technicians Tim Dunstan (University of Hull) and John Harrington (University of Leeds) with their assistance during this project.

\section{Conflicts of interest}

There are no conflicts to declare.

\section{ORCID IDs}

Paul J. Weldrick:

0000-0002-1791-5659

Matthew J. Hardman: $\quad$ 0000-0002-6423-5074

Vesselin N. Paunov: $\quad$ 0000-0001-6878-1681 


\section{References}

1. S. Leekha, C. L. Terrell, and R. S. Edson, Mayo Clinic Proc., 2011, 86 156-167.

2. K. C. Peach, W, M. Bray, D. Winslow, P. F. Linington, and R. G. Linington, Mol. bioSys., 2013, 9, 1837-1848.

3. M. A. Kohanski, D. J. Dwyer and J. J. Collins, Nat. Rev. Microbiol., 2010, 8, 423-435.

4. J. Davies and D. Davies, Microbiol. Mol. Biol. Rev. : MMBR, 2010, 74, 417-433.

5. C. Llor and L. Bjerrum, Therapeutic Advances in Drug Safety, 2014, 5, 229-241.

6. L. L. Silver, Clinic. Microbiol. Rev., 2011, 24, 71-109.

7. S. Doron and L. E. Davidson, Mayo Clinic Proc., 2011, 86, 1113-1123.

8. J. M. Munita and C. A. Arias, Mechan. Microbiology Spectrum, 2016, 4.

9. J. Davies and G. Wright, Trends in Microbiol., 1997, 5, 234-239.

10. C.P. Harkins, B. Pichon, M. Doumith, J. Parkhill, H. Westh, A. Tomasz, H. De Lencastre, S. D. Bentley, A. M. Kearns and M. T. G. Holden, Genome Biol., 2017, 18, 130.1-11.

11. S. Gardete and A. Tomasz, J. Clin. Investig., 2014, 124, 2836-2840.

12. L. Assis, M. Nedeljković and A. Dessen, Drug Resist. Updates, 2017, 31, 1-14.

13. G.G. Perron, A. E. G. Lee, Y. Wang, W. E. Huang and T. G Barraclough, Proc. Roy. Soc. B: Biol. Sci., 2012, 279, 1477-1484.

14. I. Chopra and M. Roberts, Microbiol. Mol. Biol. Reviews, 2001, 65, 232260.

15. K. Poole, Front. in Microbiol., 2011, 2, 65.1-13.

16. L. J. Markley and T. A. Wencewicz, Front. in Microbiol., 2018, 9, 1058.

17. W. Li, G. C. Atkinson, N. S. Thakor, Ü. Allas, C. Lu, K. Y. Chan, T. Tneson, K. Schulten, K. S. Wilson, V. Hauryliuk and J. Frank, Nat. Commun., 2013, 4, 1477.1-8

18. A. J. MacLeod, H. B. Ross, R. L. Ozere, G. Digout and C. E. van Rooyen, Canadian Med. Assoc. J., 1964, 91, 1056-1060.

19. D. Matzov, Z. Eyal, R. I. Benhamou, M. Shalev-Benami, Y. Halfon, Krupkin, E. Zimmerman, H. Rozenberg, A. Bashan, M. Fridman and A. Yonath, Nucl. Acids Res., 2017, 45, 10284-10292.

20. L. Spizek and T. Rezanka, Appl. Microbiol. Biotechnol., 2004, 64, 455464.

21. W. Hong, J. Zeng and J. Xie, Acta Pharmac. Sinica. B, 2014, 4, 258-265.

22. R. Leclercq and P. Courvalin, Antimicrob. Agents and Chemotherapy, 2002, 46, 2727-2734.

23. A. Fuentes-Hernandez, J. Plucain, F. Gori, R. Pena-Miller, C. Reding, G. Jansen, H. Schulenburg, I. Gudeli and R. Beardmore, PLoS Biol., 2015, 13, e1002104.

24. M. A. Wambaugh, V. P. S. Shakya, A. J. Lewis, M. A. Mulvey and J. C. S. Brown, PLoS Biology, 2017, 15, e2001644.

25. D. H. Ayhan, Y. T. Tamer, M. Akbar, S. M. Bailey, M. Wong, S. M. Daly, D. E. Greenberg and E. Toprak, PLoS Biology, 2016, 14, e1002552.1-18.

26. F. U. Din, W. Arman, L. Ullah, O. S. Qureshi, O. Mustapha, S. Shafique and A. Zeb, A. Internat. J. Nanomed., 2017, 12, 7291-7309.

27. X. Yu, I. Trase, M. Ren, K. Duval, X. Guo and Z. J. Chen, Nanomater., 2016, 1087250.

28. A. Kumari, R. Singla, A. Guliani and S. K. Yadav, EXCLI Journal, 2014, 13, 265-286.

29. H. Jahangirian, E. G. Lemraski, T. J. Webster, R. Rafiee-Moghaddam and Y. Abdollahi, Intern. J. Nanomed., 2017, 12, 2957-2978.
30. K. S. Soni, S. S. Desale and T. K. Bronich, J. Control. Rel., 2016. 240, 109-126.

31. M. Al-Awady, A. Fauchet, G. Greenway and V. J. Paunov, Mater. Chem. $B, 2017$, 5, 7885-7897.

32. M. Al-Awady, P. J. Weldrick, M. J. Hardman and V. J. Paunov, V. Mater. Chem. Front., 2018, 2, 2032-2044.

33. S.S.M Al-Obaidy, G.M. Greenway and V.N. Paunov, Nanoscale Adv., 2019, Advance article, DOI: 10.1039/c8na00121a.

34. EUCAST Breakpoint Tables v 8.0 \& v 8.1, 2018. http://www.eucast.org/clinical breakpoints/

35. G. Mi, D. Shi, M. Wang and T.J. Webster, Adv. Healthcare Mater., 2018, 7, 1800103.1-23.

36. J.T. Seil and T.J.Webster, Int. J. Nanomedicine. 2012, 7, 2767-2781.

37. B. Li and T.J. Webster, J. Orthop Res., 2018, 36, 22-32.

38. B.M. Geilich, A.L. van de Ven, G.L. Singleton, L.J. Sepúlveda, S. Sridhar and T.J. Webster, Nanoscale, 2015, 7, 3511-3519.

39. T. J. Silhavy, D. Kahne. D, and S. Walker, Cold Spring Harbor perspectives in biology, 2010, 2, a000414.

40. A. Gupta, S. Mumtaz, C.-H. Li, I.Hussain and V.M. Rotello, Chem. Soc. Rev., 2019, 48, 415-427.

41. A. Bajpai and A. Mishra, Polymer Int.., 2005, 54, 1347-1356. 